\title{
Transcriptome-based analysis of the hormone regulation mechanism of gender differentiation in Juglans mandshurica Maxim
}

\author{
Baiting Qin ${ }^{1,2}$, Xiujun Lu ${ }^{1}$, Xiao mei Sun ${ }^{1}$, Jianguo Cui ${ }^{1,2}$, Jifeng Deng ${ }^{1,2}$, Lijie Zhang ${ }^{\text {Corresp. } 1,2}$ \\ ${ }^{1}$ College of Forestry, Shenyang Agricultural University, Shenyang, China \\ 2 Key Laboratory of forest tree genetics, breeding and Cultivation of Liaoning Province, shenyang, China \\ Corresponding Author: Lijie Zhang \\ Email address: Zlj330@syau.edu.cn
}

Juglans mandshurica Maxim is a hermaphroditic plant belonging to the genus Juglans in the family Juglandaceae. The pollination period of female flowers is different from the loose powder period of male flowers on the same tree. In several trees, female flowers bloom first, whereas in others, male flowers bloom first. In this study, male and female flower buds of $J$. mandshurica at the physiological differentiation stage were used. Illumina-based transcriptome sequencing was performed, and the quality of the sequencing results was evaluated and analyzed. A total of 138,138 unigenes with an average length of $788 \mathrm{bp}$ were obtained. There were 8,116 differentially expressed genes (DEGs); 2,840 genes were upregulated, and 5,276 genes were downregulated. The DEGs were classified by Gene Ontology and analyzed by Kyoto Encyclopedia of Genes and Genomes. The signal transduction factors involved in phytohormone synthesis were selected. The results displayed that ARF and SAUR were expressed differently in the auxin signaling pathway. Additionally, DELLA protein (a negative regulator of gibberellin), the cytokinin synthesis pathway, and A-ARR were downregulated. On April 2nd, the contents of IAA, GA, CTK, ETH and $S A$ in male and female flower buds of two types of J. mandshurica were opposite, and there were obvious genes regulating gender differentiation. Overall, we found that the sex differentiation of $J$. mandshurica was related to various hormone signal transduction pathways, and hormone signal transduction plays a leading role in regulation. 


\section{Transcriptome-based analysis of the hormone} 2 regulation mechanism of gender differentiation in 3 Juglans mandshurica Maxim

${ }^{1}$ College of Forestry, Shenyang Agricultural University, Shenyang 110866, China; qinbt1828@sina.com(B.Q.); 1xjsyau@126.com(X.L.); xiaomei7280@126.com(X.S.); cjgsyau@163.com(J.C.); dengjifeng1984@hotmail.com(J.D.);

${ }^{2}$ Key Laboratory of forest tree genetics, breeding and Cultivation of Liaoning Province, Shenyang, 110866 Liaoning, China

Corresponding Author:

Lijie Zhang ${ }^{1,2}$

120 Dongling Road, Shenyang,Liaoning 110866, China

Email address: Zlj330@syau.edu.cn

\section{Abstract}

Juglans mandshurica Maxim is a hermaphroditic plant belonging to the genus Juglans in the family Juglandaceae. The pollination period of female flowers is different from the loose powder period of male flowers on the same tree. In several trees, female flowers bloom first, whereas in others, male flowers bloom first. In this study, male and female flower buds of J. mandshurica at the physiological differentiation stage were used. Illumina-based transcriptome sequencing was performed, and the quality of the sequencing results was evaluated and analyzed. A total of 138,138 unigenes with an average length of $788 \mathrm{bp}$ were obtained. There were 8,116 differentially expressed genes (DEGs) (TSA SUB10193123); 2,840 genes were upregulated, and 5,276 genes were downregulated. The DEGs were classified by Gene Ontology and analyzed by Kyoto Encyclopedia of Genes and Genomes. The signal transduction factors involved in phytohormone synthesis were selected. The results displayed that ARF and SAUR were expressed differently in the auxin signaling pathway. Additionally, DELLA protein (a negative regulator of gibberellin), the cytokinin synthesis pathway, and A-ARR were downregulated. On April 2nd, the contents of IAA, GA, CTK, ETH and SA in male and female flower buds of two types of $J$. mandshurica were opposite, and there were obvious genes regulating gender differentiation. Overall, we found that the sex differentiation of J. mandshurica was related to various hormone signal transduction pathways, and hormone signal transduction plays a leading role in regulation.

\section{Introduction}

Heterosexualism is a transitional type in the evolution of plants from monoecious to dioecious. 
40 reproductive system has been differentiated by male and female flowers to form two mating

41 types, namely, female and male flowers (Gleiser $\mathrm{G}$ et al., 2008; Teichert et al., 2011; Wang et al., 42 2012; Fukuhara et al., 2014; Renner SS, 2014; Sun et al., 2016). The dioecious mating system is

43 not common in flowering plants (Wang, 2001). At present, only Batulaceae, Corylus,

44 Juglandaceae, Carya, Juglans, and Cyclocarya are woody plants with a monoecious mating

45 system. Other Acer species of Aceraceae belong to bisexual flowers, but they are unisexual or 46 monoecious in function. There are many patterns of flower expression sequences in dioecious 47 plants, but generally speaking, these two mating types are fixed (Liu,2016; Fu et al., 2010; Tal, 48 O,2009), and in most species of Juglans, the ratio of males to females in the mating-type is 1:1.

49 Sex-determining genes are the genetic basis of sex differentiation, and hormones play an

50

51

52

53

54

55

56

57

58

59

60

61

62

63

64

65

66

67

68

69

70

71

72

73

74

75

76

77

78

79 important role in regulating flower sex differentiation as induction signals in the process of plant sex differentiation (Golenberg and West, 2013; Aryal et al., 2014; Kong,2009). These endogenous hormones regulate flower induction and formation through various flowering pathways (photoperiod pathway, gibberellin pathway, spring flower pathway, and autonomous pathway) (Mutasa Gottgens and Hedden, 2009; Amasino R M, 2010; Lee et al., 2010; Xing et al., 2015, 2016; Shimakawa et al., 2012). Molecular studies have revealed that plant hormones are one of the induction signals of plant gender differentiation and play an important role in regulating the process of gender differentiation (Tanurdzic and Banks, 2004; Kong,2009).

However, there is a complex interaction among the hormones that makes the regulation mechanism of plant hormones on gender determination and differentiation extremely complex, and there is no unified regulation model (Li, 2016; Yu et al., 2020; Zhong and Qin, 2010). The same hormone may lead to the expression of different gender differentiation programs in different plants through the difference in the signal transduction process (Xing, 2007; Wang, 1996; 1997; Chen, 2005; Anusak et al., 2003; Arora et al., 2007). To date, the control theory of hormone-regulating sex determination has focused on several main economic plants but has not been reported in dioecious woody plants. Research on the flowering mechanism of dioecious woody plants is limited to the basic research on cytohistology and molecular biology of male and female flower bud differentiation.

Juglans mandshurica is a broad-leaved tree species of Juglans in Juglandaceae. It is also an important woody tree species for grain and oil and an economic tree species for both fruit and wood in China (Zhang et al., 2019a). Juglans mandshurica fruit and wood have broad prospects for development and utilization. In recent years, a research group collected and investigated the germplasm resources of $J$. mandshurica in Northeast China and found that $J$. mandshurica has monoecious heteromorphism, and there are mainly two mating types: protandrous and protogynous. The male inflorescence of protandrous is synchronized with the spreading leaf, and the female flower begins to bud after the male flower matures and scatters; the development of female flowers of protogynous is synchronous with leaf spreading, while the development of male flowers is slow. After the pollination of female flowers is complete, the male flowers grow rapidly and mature and scatter pollen. The fertile period of female flowers is different from the scattering period of male flowers. The flowering sequence of males and females is very stable, 
80

81

82

83

84

85

86

87

88

89

90

91

92

93

94

95

96

97

98

99

100

101

102

103

104

105

106

107

108

109

110

111

112

113

114

115

116

117

118

119

and the two mating types are randomly distributed in the population (Zhang et al., 2019b). This reproductive characteristic leads to a lack of flowering time between male and female flowers, which seriously affects the pollination rate and fruit setting rate. Therefore, in this study, the molecular mechanism of hormone regulation in the process of sexual differentiation of $J$. mandshurica was analyzed using high-throughput Illumina transcriptome sequencing technology, and the interaction between sexual differentiation and endogenous hormone synthesis and signal transduction was explored. The results of this study can be used to guide forestry production, and provide an effective theoretical basis and technical support for improving the seed yield and quality of J. mandshurica by rationally allocating the proportion of different mating types of plantation populations and manually controlling pollination.

\section{Materials \& Methods}

\section{Plant Material}

Based on the previous research results of the phenology, morphology, physiology, and cytology of $J$. mandshurica natural forest in eastern Liaoning, China, the morphological development period, physiological differentiation period, and vegetative growth period of $J$. mandshurica flower bud differentiation process were determined. In 2019, the flower buds were selected on March 17 and 25, and April 2, 10, and 15 to be quick-frozen in liquid nitrogen and stored in a refrigerator at $-80^{\circ} \mathrm{C}$ for differential gene expression analysis and metabolomics.

Simultaneously, the female and male flower buds collected on April 2 during the physiological differentiation period of the male and female precursors (Figure 1) were selected for transcriptomic research. The female flower buds of the female precursor type were named T1, which were repeated three times, namely A1, A2, and A3, respectively. The male flower buds were named T2, which were repeated three times, namely A4, A5, and A6. The female flower buds of the male precursor type were named M1, which were repeated three times, namely B1, B2, and B3. The male flower buds were named M2, which were repeated three times, namely B4, B5, and B6. There were 12 samples in total.

\section{Construction and Sequencing of Library}

Oligo(dT) magnetic beads were used to enrich mRNA with polyA structure in total RNA, and the RNA was interrupted to 200-300 bp fragments by ion interruption. After the construction of the library was completed, PCR amplification was adopted to enrich the library fragments, and then library selection was carried out according to the fragment size, where the library size is 300-400 bp. Next, the library was inspected using an Agilent 2100 Bioanalyzer, and the total concentration and effective concentration of the library were detected. After RNA extraction, purification, and library building, these libraries were sequenced using the second nextgeneration sequencing technology based on an Illumina HiSeq sequencing platform. The sequencing raw read length was $150 \mathrm{bp}$, and the original sequence of mRNA in the physiological differentiation stage of male and female flower buds of J. mandshurica was obtained.

\section{Sequencing Data Sorting and Transcript Assembly}

We counted the Raw Data of each sample separately, spliced Clean Reads with Trinity software to obtain transcripts, and then carried out the subsequent analysis. Trinity was used for De novo 
120 assembly software for transcriptome splicing, which splices high-quality sequences based on the 121 De Bruijn Graph splicing principle. After splicing was completed, the transcript sequence file in 122 the FASTA format was obtained. The longest transcript under each gene was extracted as the 123 representative sequence of the gene, called unigene.

124 Unigene Annotation Classification and Expression Analysis

125 The database used for gene function annotation included NR (NCBI non-redundant protein 126 sequences), GO (Gene Ontology), KEGG (Kyoto Encyclopedia of Genes and Genome), eggnog 127 (evolutionary genealogy of genes: Non-supervised Orthologous Groups), and Swiss-Prot.

128 Differential Expression and Enrichment Analysis

129 By comparing the RNA-seq data of the physiological differentiation stage of female and male 130 flower buds, the gene expression was analyzed by DESeq. The conditions for screening 131 differentially expressed genes were as follows: expression difference multiple |log2FoldChange| $132>1$, significant P-value $<0.05$. Significant enrichment analyses were conducted of differentiated genes based on the number of differentially expressed genes contained at different levels of each KEGG pathway, after multiple tests and corrections, the pathway with a Q-value $\leqslant 0.05$ was selected as the pathway significantly enriched in differentially expressed genes, and then the metabolic pathway and signal pathway in which differentially expressed genes were mainly 137 involved were determined. qRT-PCR Verifies Sequencing Data Twelve differential expression genes were randomly selected, and their expression profiles were detected via qRT-PCR to verify transcriptome data.

\section{Endogenous Hormone Determination}

Samples from different periods were tested for phytohormones, including IAA, GA, CTK, ABA, ETH, BR, JA, and SA, then analyzed and compared (Weiler et al., 1981).

145

146

\section{Transcriptome Sequencing Analysis of Male and Female Flower Buds} Raw transcriptome sequencing reads all flower bud samples with raw sequencing reads of more than $40 \mathrm{M}$, an average reading length of $150 \mathrm{bp}$, a total number of sequencing bases of over $6 \mathrm{G}$ nt, a Q20 content of over 95\%, and a Q30 content of over 90\%. Statistical analysis revealed that the sequencing data were of high quality and could meet the requirements of further analysis. The data filtering used Cutadapt to remove the 3'-end linker, and the removed part had at least 10 bp overlap (AGATCGGAAG) with the known linker, allowing a 20\% base mismatch. Remove read with an average mass fraction lower than Q20. The number of clean reads obtained from female flower buds of female precursor type ranged from 43894726 to 46469762 . The number of high-quality sequence reads was 6610627884 to 6991929010 , the percentage of high-quality sequence reads in sequencing reads was $99.41 \%-99.49 \%$, and the percentage of high-quality sequence bases in sequencing bases was $99.13 \%-99.16 \%$ (Table S1). contig were obtained according to the overlap of reads, and these contigs were linked together by overlap to obtain unigene. Transcript and unigene sequences were 344218 and 138138 , 
160

161

162

163

164

165

166

167

168

169

170

171

172

173

174

175

176

177

178

179

180

181

182

183

184

185

186

187

188

189

190

191

192

193

194

195

196

197

198

respectively, and the transcript and unigene sequences had average lengths of $1182 \mathrm{bp}$ and 788 $\mathrm{bp}$, respectively. The number of unigene in the physiological differentiation stage of male and female flower buds of $J$. mandshurica was approximately 80000 , while that of unigene larger than $500 \mathrm{bp}$ was almost $60 \%$ of the total. This indicated that the sequencing results were of high splicing quality, which was conducive to further functional annotation analysis (Figure 2). All-unigene obtained by transcriptome sequencing was annotated with protein and nucleic acid functions (Table 1). Among the 108828259 bp unigene assembled in total, all-unigene sequences were sequentially aligned to the protein database through blastx, and unigene was annotated with gene functions. The databases used for gene function annotation included NR (NCBI NonRedundant Proteins Sequences), GO (Gene Ontology), KEGG (Kyoto Encyclopedia of Genes and Genome), eggNOG (evolutionary genealogy of genes: Non-supervised Orthologous Groups), and Swiss-Prot. The number of successful annotations of unigene into the Nr protein database reached 60415 , accounting for $43.74 \%$ of the total number of all-unigene. The number of unigenes annotated in the GO database reached 29402, accounting for $21.28 \%$ of the total unigenes. The number of unigenes annotated in the eggNOG database reached 57250, accounting for $41.44 \%$ of the total unigenes. The number of unigenes annotated in the Swissprot database reached 45347 , accounting for $32.83 \%$ of the total unigenes, while the number of unigenes annotated in the KEGG database was at least 6845 , accounting for only $4.96 \%$ of the total. The number of unigenes annotated in all databases was 5312, accounting for only $3.85 \%$ of the total unigenes.

According to the statistics of unigenes annotated in the $\mathrm{Nr}$ protein database by E-value distribution, e-values in $1 \mathrm{e}^{-5}-1 \mathrm{e}^{-15}$ accounted for $26.72 \%$, and $1 \mathrm{e}^{-15}-1 \mathrm{e}^{-30}$ accounted for $22.33 \%$ (Figure S1a). According to the annotation similarity distribution statistics, $19.45 \%$ of the sequences were concentrated in the $80 \%-90 \%$ range, with a total of $55.51 \%$ sequence similarity higher than $60 \%$ (Figure S1b). Homology analysis among different species demonstrated that Vitis vinifera had the highest homology, reaching 19.26\%, followed by Ziziphus jujuba (6.29\%), and other species (58.27\%). The distribution of species homology revealed high homology with grape (Figure S1c).

The gene was annotated by eggNOG comparison, and the eggNOG number of the best comparison result was assigned to the corresponding gene. Further, using the correspondence between the eggNOG number and the eggNOG classification catalog, each gene was classified into the eggNOG classification catalog. A total of 77173 unigenes were compared to the eggNOG database (Figure 3). Among them, 21187 were compared with the predicted general function prediction only, and 15111 were compared with the predicted unknown function. Then, compared with the predicted number of aggregation classifications, the sequence was 4728 signal transmission mechanisms, 4354 transcriptions, 4069 replications, recombination, and repair. Post-translational modification, protein transformation, 4042 molecular chaperones, 2896 translations, ribosome structure, and biogenesis. The above approach demonstrated that substance and energy metabolism were very vigorous in the process of $J$. mandshurica flower 
199 bud differentiation, cell growth, proliferation, and division speed were very fast, and hormone 200 signal transduction participated in the differentiation process.

201 GO annotation was completed using BLAST2 GO software, and the annotation adopted the 202 default parameters of BLAST2 GO. The results of the GO annotation were mapped to go term, 203 and the number of genes annotated in the second-level classification was counted (Figure S2).

204 According to classification statistics, there were 29402 (21.8\%) unigene genes annotated in the $J$. 205 mandshurica transcription group into the GO database. There were 3 major categories of 206 annotations: 32959 (24.5\%) of molecular function, 51672 (38.4\%) of biological processes, and 20750032 (37.2\%) cellular components. There were 67 functional groups. Among the 24 functional 208 groups of biological processes, unigene included 14613 metabolic processes (GO: 0008152) and 209

210

211

212

213

214

215 14514 cellular processes (GO: 0009987). There were 10152 cells (GO: 005623) and 10063 cells part (GO: 0044464). Among the 19 functional groups of molecular functions, there were 14843 bindings (GO: 0005488) and 14061 catalytic activities (GO: 0003824). Through GO function annotation, a large number of unigenes related to metabolic process, biological regulation, cell process, cell, membrane, and membrane component organelles, and other unigenes were obtained in the three major types of functions. These genes can provide a theoretical basis for the study of sex differentiation and flowering related functional genes of $J$. mandshurica.

216 A total of 6288 unigenes were mapped to 35 KEGG metabolic pathways, of which 606 genes

217 were annotated by the signal transduction pathway in environmental information processing. A

218

219 total of 504 genes were annotated by the translation pathway in genetic information processing, and 487 genes were annotated by carbohydrate metabolism in the metabolic pathway (Figure 220 S3).

221

222

223

224

225

226

227

228

229

230

231

232

233

234

235

236

237

238

\section{Analysis of GO and KEGG Pathway in Male and Female Flower Buds}

According to the classification and annotation of the GO function of differentially expressed genes in male and female flower buds of female precursor type (T1vsT2), we can observe that there were 837 functions, including 230 molecular functions, 517 biological processes, and 90 cell compositions. We analyzed the significance of $\mathrm{GO}$ function enrichment of female and male flower buds of female precursor type (when Bonfeeoni-corrected P-value $\leqslant 0.05$ ). The results revealed that T1vsT2 significantly enriched 36 terms, 18 terms belonged to the biological process, 8 terms belonged to molecular function, and 10 terms belonged to cellular component (Figure 4). The enriched term included nucleic acid binding transcription factor activity (GO: 0001071), regulation of RNA biosynthesis processes (GO: 2001141), ribosomal subunit (GO: 0044391 ), etc. The cluster frequencies are $6.1 \%, 6.7 \%$, and $9.9 \%$, respectively. The main annotated genes include $G A I, S O C 1, A P 1$, etc. The metabolism of endogenous substances changed during the physiological differentiation of female and male flower buds of $J$.

mandshurica, and, simultaneously, the genes related to flower formation were pre-activated to a certain extent, laying a foundation for the differentiation of flower organs. There were 121 genes annotated on the pathway compared in T1 vs T2. Differential Expression Genes (DEGs) significantly enriched 20 pathways in KEGG (Figure 5), of which plant hormone signal transduction was the highest (ko04075). The second was carbon metabolism (ko01200). 
239 Additionally, glyoxylate and dicarboxylate metabolism (ko00630), AMPK signaling pathway

240 (ko04152), and zeatin biosynthesis (ko00908) were also significantly enriched. These results

241 demonstrate that hormones play a leading role in regulating the differentiation of female and

242 male flower buds of the female precursor type, and carbohydrate was also an essential metabolic

243 substance during the transformation from physiological differentiation of flower buds to

244 morphological differentiation.

245 Male and female flower buds of male precursor type (M1 vs M2) had a total of 1013 functions

246 annotated, including 649 biological processes, 94 cell compositions, and 270 molecular functions

247 (Figure 6). 54 terms were significantly enriched, included 28 belonged to biological process, 21

248 belonged to molecular function and 5 belonged to cellular components. The enriched term

249 included protein dimerization activity (GO: 0046983), regulation of transcription, DNA-

250 templated (GO: 0006355), extracellular region (GO: 0005576), etc. The cluster frequencies are

$2515.6 \%, 9.8 \%$, and $4.0 \%$, respectively. The main annotated genes include ARF5, GA2OXI, TEM1,

252 SOC1, etc. A total of 125 differentially expressed genes DEGs of M1 vs M2 were enriched in the

253 KEGG pathway (Figure 7). Among them, the ribosome (ko03010) was the most abundant,

254 followed by plant hormone signal transmission (ko04075) and carbon metabolism (ko01200),

255 which indicated that M1 vs M2 was affected by gene regulation, hormone regulation, and

256 physiological metabolites in the process of sex differentiation. The KEGG significant enrichment

257 analysis (Bonferroni corrected P-value $\leqslant 0.05$ ) revealed that the KEGG of M1 vs M2

258 significantly enriched the 18 pathways. Among them, plant hormone signal transduction

259 (ko04075) was the highest, followed by the neurotrophin signaling pathway (ko04722) and

260 starch and sucrose metabolism (ko00500). This indicates that hormones play a leading role in the

261 physiological differentiation of male and female flower buds of male precursor type and

262 regulated the growth and differentiation of organs in all parts of the flower.

\section{Analysis of DEGs}

264 According to the screening of differentially expressed genes in the flower bud transcriptome of $J$.

265 mandshurica, we obtained the DEGs comparison library of female and male flower buds: male

266 pre-type female flower buds and male flower buds (M1 vs M2, 1357 genes), male pre-type

267 female flower buds and female pre-type female flower buds (M1 vs T1, 1461 genes), male pre-

268 male flower buds and female pre-type male flower buds (M2 vs T2, 3476 genes), female pre-

269 type female flower buds, and male flower buds (T1 vs T2, 1822 genes) (Figure 8A). This

270 indicated that most of the DEGs appeared in flower buds of different genders, and 11 of them

271 were differentially expressed in the four libraries. 8116 differentially expressed genes were

272 obtained in the four cDNA libraries, including 2840 upregulated genes and 5276 downregulated

273 genes (total 8116 genes) in Figure 8A and 8B. The downregulated genes in male and female

274 flower buds of the two mating types were generally higher than those of the upregulated genes.

275 In M1 vs M2, 585 genes were upregulated and 772 genes were downregulated. In T1 vs T2, 359

276 genes were upregulated and 1463 genes were downregulated (Figure 8B) (Data related to

277 differential expressed genes have been submitted to NCBI database and TSA database, TSA

278 SUB10193123). 
279 In the signal transduction and synthesis pathways of plant hormones, we compared the 280 differentially expressed genes in M1 vs M2 and T1 vs T2 libraries and found that the sex 281 differentiation of flower buds was mainly related to ARF, SAUR, DELLA, TF, PR-1, etc (TSA 282 SUB10193123, also uploaded as the supplementary file, Data related to differential expressed 283 genes. zip).

284 Differential Expression of TFs in male and female flower buds

285 A total of 195 TFs were differentially expressed in M1 vs M2, 168 TFs were differentially 286 expressed in T1vsT2, and 83 TFs were differentially expressed in M1vsM2 and T1vsT2 . The 287 differentially expressed TFs included members of ARF, AP2, C2H2, ERF, bHLH, MADS, 288 MYB, WRKY, NAC and other TF family members were included. MADS and bHLH families

289

290

291

292

293

294

295

296

297

298

299

300

301

302

303

304

305

306

307

308

309

310

311

312

313

314

315

316

317

318 were dominant among the families. We have screened out many transcription factors related to plant hormones, and based on the results, we have identified transcription factors with differential expression in auxin and ethylene signaling pathways (Figure 9). There were eight identical differentially expressed transcription factors in M1 vs M2 and T1 vs T2.

\section{Verification of Gene Expression via Real-time PCR}

To verify the gene expression pattern identified by RNA-Seq data, the transcription levels of 12 randomly selected DEGs were detected by quantitative PCR. We used these 12 genes for qRTPCR, with 3 biological replications in each group. The gene expression pattern detected by qRTPCR was consistent with the RNA sequence data (Figure 10). The qRT-PCR experiments confirmed that the DEGs obtained from the assembled transcriptome were accurate and the gene expression profile obtained from RNA-Seq data was reliable.

\section{Determination of Endogenous Hormones in Flower Buds of $\boldsymbol{J}$. mandshurica}

To explore the relationship between the flower bud differentiation of $J$. mandshurica and plant endogenous hormones, we determined the content of 8 plant hormones in flower buds at the early stage of physiological differentiation. The content of plant endogenous hormones affected flowering time. By measuring the plant hormones in the male and female flower buds of $J$. mandshurica at the early stage of physiological differentiation (Table 2), we found that the contents of auxin (IAA), brassinolide (BR), and salicylic acid (SA) demonstrated an overall upward trend. Gibberellin (GA) content first increased and then decreased, and a peak appeared on approximately April 10th. The content of cytokinin (CTK) fluctuated: up-down-up. The contents of ethylene (ETH) and abscisic acid (ABA) first decreased and then increased. Jasmonic acid (JA) content in flower buds of female precocious $J$. mandshurica was in a relatively stable state before April 10th and began to increase after this date, while JA content in flower buds of male precocious $J$. mandshurica revealed an overall upward trend. Interestingly, on April 2nd, the contents of IAA, GA, CTK, ETH, and SA were all found in the flower buds of $J$. mandshurica: $\mathrm{T} 1<\mathrm{T} 2$ and $\mathrm{M} 1>\mathrm{M} 2$. The female flowers of the female pre-type $J$. mandshurica open first, and the male flowers disperse pollen later. The male flowers of the male pre-type $J$. mandshurica are scattered first, and the female flowers open later. The contents of the above five hormones in the male and female flower buds of the two types of J. mandshurica were opposite, indicating that there were genes regulating gender differentiation in these five hormones, which 
was consistent with the differential expression of genes in the transcriptome data. We inferred that these hormones participate in gender differentiation to varying degrees and that there may be interactions. BR and JA contents were as follows: $\mathrm{T} 1<\mathrm{T} 2$ and $\mathrm{M} 1<\mathrm{M} 2$. The contents of BR and JA in female flower buds were lower than those in male flower buds, indicating that the regulation of these two hormones was more important in male flower buds. ABA content revealed that: $\mathrm{T} 1>\mathrm{T} 2$ and $\mathrm{M} 1>\mathrm{M} 2$, while the $\mathrm{ABA}$ content in female flower buds was higher than that in male flower buds, indicating that $\mathrm{ABA}$ regulation was more needed in female flower buds.

\section{Discussion}

Plant endogenous hormones are involved in the regulation of gender differentiation in almost all plants. However, it is generally believed that CTK and GA are the main hormones that affect gender differentiation in plants, while other hormones affect gender expression by changing the activities of GA and CTK (Liu et al., 2016). In this study, according to the sequencing analysis of the flower bud transcriptome of $J$. mandshurica, it was found that the process of sexual differentiation of $J$. mandshurica is a complex regulatory network composed of many factors. Furthermore, it is influenced by flowering gene regulation, hormone regulation, and physiological and biochemical metabolites in the process of sexual differentiation. Additionally, hormone played a leading role in the physiological differentiation of male and female flower buds, regulating the growth and differentiation of various organs of flowers. This is consistent with the results of Wu et al. (2010) who found that cucumber sex differentiation genes participate in the signal transmission of plant endogenous hormones by analyzing the differences in transcripts of cucumber plants of different genders. The genes include ACS, ASR1, CSIAA2, CSAUX1, and other genes constituting a regulatory network that works together to regulate cucumber sex differentiation. These plant hormones include GA, ABA, ETH, IAA, CTK, and BR. Additionally, SA, JA, and other plant hormones play key roles in the process of gender differentiation (Conti L, 2017; Yamaguchi et al., 2014; Nakagawa et al., 2012; Liron S et al., 2014; Papadopouloue et al., 2005; Pan et al., 2014; Chen et al.,2017; Zhu et al.,2015;

Khryanin,2002). The AUX, A-ARR, DELLA, TF, PYL, ERF1/2, CYCD3, and PR-1, which have significant regulatory effects on the flower bud differentiation of $J$. mandshurica, are classified into IAA, GA, ETH, CTK, ABA, BR, and SA synthesis pathways, and they work together to regulate hermaphroditism. Teo et al. (2014) found that IAA plays an indispensable role in the regulation of inflorescence differentiation in A. thaliana, and the interference of IAA synthesis, transport, and signal transduction leads to pistil or stamen defects. Aryal et al. (2014) found that high expression of miRNAs on male flowers was related to IAA signaling in the study of sex differentiation of Carica papaya. However, ARF, SAUR, PYL, PLR, and other genes were significantly expressed in the signaling pathway of IAA and ABA in the male and female flower buds of $J$. mandshurica, indicating that these two plant hormones could regulate the differentiation of male and female flower buds. The endogenous GA3, ZT and ABA of loquat (Eriobotrya japonica) flower were determined during its development, and it was found that there was a high correlation between the change of endogenous hormone concentration and the 
359

360

361

362

363

364

365

366

367

368

369

370

371

372

373

374

375

376

377

378

379

380

381

382

383

384

385

386

387

388

389

390

391

392

393

394

395

396

397

398

expression level of genes related to hormone signal transduction pathway (Jing et al.,2020). In addition, transcriptome analysis of Madhuca pasquieri showed that auxin, GA, ABA and cytokinin metabolism pathway were related to seed germination and post germination (Kan et al.,2020). ETH also plays an important role in the gender differentiation of plants. Duan et al. (2008) found that the increase in ETH content affected the development of stamens during the flower bud differentiation of $A$. thaliana, and stamens were most sensitive to ETH in all flower organs. ERF was downregulated in the ETH signaling pathway of male flower buds of $J$. mandshurica. The expression levels in female flower buds and male flower buds are different. In the determination results of ETH, we found that the ETH content was at a low level before April 2, and began to increase greatly after April 2, which may indicate that ETH played an important role in the physiological differentiation of flower buds. Additionally, the change trend of ABA content is similar to that of ETH, which may have synergistic effect. The most important physiological function of CTK is to induce bud differentiation, its specific role in flower bud differentiation is not clear, but it is known that CTK is involved in regulating the cell division and differentiation of flower meristems (Jacqmard et al. 2003). Nibau et al. (2011) found that SUP, a regulatory gene for flower organ development, controls the development of male and female flowers by acting on CTK signal transduction. In the process of flower bud differentiation of $J$. mandshurica, A-ARR was downregulated in the CTK signal transduction pathway of male flower bud, but there was no differential expression in a female flower bud, indicating that AARR may be involved in the flower bud gender differentiation of J. mandshurica. Wang (2019) found that BR can participate in many processes of regulating plant growth and development, but also interacts with other hormones, interacting with the IAA signal pathway through ARF. However, the CYCD3 gene was upregulated in the BR signaling pathway in female flower buds of $J$. mandshurica, but it was not differentially expressed in male flower buds. SA is a negative regulator of FLC and other flowering inhibition genes in the autonomous flowering pathway (Wada et al., 2010), and the PR-1 gene in the SA signaling pathway in the flower buds of $J$. mandshurica is downegulated, which may also have a certain influence on the flowering of $J$. mandshurica. TFs play an important role in the reproductive development of $J$. mandshurica. Studies have demonstrated that TFs play an important role in the sex determination and differentiation of plants and animals. In our study, many TF genes were expressed differently in the male and female flower buds of $J$. mandshurica. In different TF families, bHLH, MADS, NAC, MYB, and ARF are significantly enriched, indicating that they play an important role in the sex determination or differentiation of $J$. mandshurica. Members of these families are involved in the gender differentiation of other monoecious or monoecious plants (Akagi et al., 2014; Martin et al., 2009).By sequencing the transcripts of male and female flower buds of $J$. mandshurica, we found a large number of candidate differential genes involved in various biological processes, which may be related to the regulation of the formation of male and female flower buds. Combined with the determination results of endogenous hormone content in male and female flower buds at the early stage of physiological differentiation, it demonstrates that the change in hormone content does affect the flowering time, and the contents of IAA, BR, and SA 
399

400

401

402

403

404

405

406

407

408

409

410

411

412

413

414

415

416

417

418

419

420

421

422

423

424

425

426

427

428

429

430

431

432

433

434

435

436

437

438

generally demonstrate an upward trend during the development of flower buds. This indicates that there was a significant correlation between endogenous hormone levels in plants and gender differentiation, which was consistent with the research results of Zhong and Qin (2010). Each plant hormone does not independently regulate the expression of plant sex, but a variety of hormones form a network regulation model to interact and influence the gender differentiation of plants. Metabolic substances and energy metabolism are very vigorous in the process of flower bud differentiation of $J$. mandshurica. Cell growth, proliferation, and division are very fast, and hormone signal transduction is involved in the process of gender differentiation. Simultaneously, it is influenced by many environmental factors and endogenous hormones, especially the signal transduction and synthesis of endogenous hormones such as IAA, GA, CTK, and SA, which are the main reasons for the emergence of two mating types of J. mandshurica in the process of flower bud sex differentiation. In order to further understand the specific biological process and gene regulation related to sex differentiation of $J$. mandshurica, we will determine the proteome and metabonomics of $J$. mandshurica flower buds and the hormone content in the leaves and fruits in the future research, and further explore the regulation mechanism of sexual differentiation in J. mandshurica.

\section{Conclusions}

In this study, it was found that participation in the sex expression of $J$. mandshurica was related to metabolic pathways such as IAA, GA, CTK, etc. Through analysis and verification, the signal transduction factors involved in plant hormone synthesis were screened. The downregulated genes in the different genes between male and female flower buds were generally higher than the upregulated genes, and many of them inhibited flowering. When the genes such as SAUR, DELLA, and A-ARR are downregulated, flowering is promoted. During the process of flower bud sex differentiation, hormones play a leading role in regulating the growth and differentiation of flower organs.

\section{Acknowledgements}

We thank the Shanghai Personalbio Biotechnology Company for assisting with the sequencing analysis. We are grateful to the editor and reviewers for their insightful comments and suggestions.

\section{References}

Akagi T, Henry IM, Comai L. 2014. A Y-chromosome-encoded small RNA acts as a sex determinant in persimmons. Science. 346:646-50.

Amasino RM. 2010. Seasonal and developmental timing of flowering. The Plant Journal. 61: 1001-1013.

Anusak P, Ditta GS, Savidge B, Liljegren SJ, Baumann E, Wisman E, Yanofsky MF. 2003. Assessing the redundancy of MADS-box genes during carpel and ovule development. Nature. 424:85-88.

Arora R, Agarwal P, Ray S, Singh AK, Singh VP, Tyagi AK, Kapoor S. 2007.MADS-box gene family in rice: genome-wide identification, organization and expression profiling during reproductive development and stress. BMC Genomics. 8:242. 
439

440

441

442

443

444

445

446

447

448

449

450

451

452

453

454

455

456

457

458

459

460

461

462

463

464

465

466

467

468

469

470

471

472

473

474

475

476

477

Aryal R, Jagadeeswaran G, Zheng Y, Yu Q, Sunkar R, Ming R. 2014. Sex specific expression and distribution of small RNAs in papaya. BMC Genomics. 15(1):119-123. https://doi:10.1186/1471-2164-15-20.

Aryal R, Ming R. 2014. Sex determination in flowering plants: papaya as a model system. Plant Science. 21, 56-62. https://doi:10.1016/j.plantsci.2013.10.018.

Chen H, Lu X, Xu L, Yi K, Tian Y. 2005.Sex Determination Genes and Sex Expression Mechanism in Cucumber. Plant physiology communication. 41(1): 7-13. https://doi:10.13592/j.cnki.ppj.2005.01.002.

Chen M, Pan B, Fu Q, Tao Y, Martínez-Herrera J, Niu L, Ni J, Dong Y, Zhao M, Xu Z. 2017. Comparative Transcriptome Analysis between Gynoecious and Monoecious Plants Identifies Regulatory Networks Controlling Sex Determination in Jatropha curcas. Frontiers in Plant Science. 7. https://doi: 10.3389/fpls.2016.01953.

Conti L. 2017. Hormonal control of the floral transition: Can one catch them all. Developmental Biology. 430(2): 288-301. https://doi:10.1016/j.ydbio.2017.03.024.

Duan Q, Wang D, Xu Z, Bai S. 2008.Stamen development in Arabidopsis is arrested by organspecific over expression of a cucumber ethylene synthesis gene CsACO2. Planta. 228(4):537-543. https://doi: 10.1007/ s00425-008-0756-7.

Fu X, Feng L, Fang S, Mao J. 2010. Observation on flowering habits and anatomy of stamen development in Cyclocarya paliurus. Journal of Nanjing Forestry University(Natural sciences edition). 34(3):67-71.

Fukuhara T, Tokumaru S. 2014. Inflorescence dimorphism, heterodichogamy and thrips pollination in Platycarya strobilacea (Juglandaceae). Annals of botany.113:467-476. https://doi:10.1093/aob/mct278

\section{Gleuser G, Verdu M, Segarra-Moragues JG, González-Martínez SC, Pannell JR.} 2008. Disassortative mating, sexual specialization, and the evolution of gender dimorphism in heterodichogamous Acer opalus. Evolution. 62(7):1676-1688.

Golenberg EM, West NW. 2013. Hormonal interactions and gene regulation can link monoecy and environmental plasticity to the evolution of dioecy in plants. American journal of botany. 100:1022-1037. https://doi:10.3732/ajb.1200544.

Jacqmard A, Detry N, Dewitte N, Onckelen HV, Bernier G. 2002.In situ localisation of cytokinins in the shoot apical meristem of Sinapis alba at floral transition. Planta. Vol.214 (6), pp.970-973.

Jing D, Chen W, Hu R, Zhang Y, Xia Y, Wang S, He Q, Guo Q, Liang G. 2020. An Integrative Analysis of Transcriptome, Proteome and Hormones Reveals Key Differentially Expressed Genes and Metabolic Pathways Involved in Flower Development in Loquat. International journal of molecular sciences. 21, 5107; https://doi:10.3390/ijms21145107.

Kan L, Liao, Q.; Su, Z.; Tan, Y.; Wang, S.; Zhang, L. 2020. Single-Molecule Real-Time Sequencing of the Madhuca pasquieri (Dubard) Lam. Transcriptome Reveals the Diversity of Full-Length Transcripts. Forests. 11, 866. https://doi:10.3390/f11080866. 
478

479

480

481

482

483

484

485

486

487

488

489

490

491

492

493

494

495

496

497

498

499

500

501

502

503

504

505

506

507

508

509

510

511

512

513

514

515

Khryanin VN. 2002. Role of phytohormones in sex differentiation in plants. Russian Journal of Plant Physiology. 49:545-551. https://doi:10.1023/A:1016328513153.

Kong D. 2009. Progress in Hormonal Regulation to Sex Differentiation in Higher Plants. Journal of Anhui Agri. Sci. 37(12):5352- 5354,5378. https://doi:10.13989/j.cnki.05176611.2009.12.054.

Lee J, Lee I. 2010. Regulation and function of SOC1, a flowering pathway integrator. Journal of experimental botany.61: 2247-2254. https://doi:10.1093/jxb/erq098.

Li J. 2016. Morphology Observation and Study on Regulation Mechanism of Phytohormones during Floral Sex Differentiation in Persimmon. China Academy of Forestry.

Liron S, Sivan S, Naftali Z, Lyudmila S, Adi DF, Eduardo B, Avi S. 2014. Fruit load induces changes in global gene expression and in abscisic acid (ABA) and indole acetic acid (IAA) homeostasis in citrus buds. Journal of experimental botany. 65:3029-3044. https://doi:10.1093/jxb/eru148.

Liu J, Mao X, Li X, Fu X. 2016. A review on flowering mechanism in heterodichogamous plants. Journal of Nanjing Forestry University (Natural Science Edition). 40(1):147-154.

Martin A, Troadec C, Boualem A, Rajab M, Fernandez R, Morin H, Pitrat M, Dogimont C, Bendahmane A. 2009. A transposon-induced epigenetic change leads to sex determination in melon. Nature. 461:1135-8.

Mutasa-Göttgens E, Hedden P. 2009. Gibberellin as a factor in floral regulatory networks. Journal of experimental botany. 60:1979-1989. https://doi:10.1093/jxb/erp040.

Nakagawa M, Honsho C, Kanzaki S, Shimizu K, Utsunomiya N. 2012.Isolation and expression analysis of FLOWERING LOCUS T-like and gibberellin metabolism genes in biennial-bearing mango trees. Scientia Horticulturae. 139: 108-117. DOI:10.1016/j.scienta.2012.03.005.

Nibau C, Di Stilio VS, Wu H, Cheung AY. 2011. Arabidopsis and tobacco SUPERMAN regulate hormone signaling and mediate cell proliferation and differentiation. Journal of experimental botany. 62(3):949-961. https://doi:10.1093/jxb/erq325.

Pan B, Chen M, Ni J, Xu Z. 2014.Transcriptome of the inflorescence meristems of the biofuel plant Jatropha curcas treated with cytokinin. BMC Genomics. 15:974. https://doi:10.1186/1471-2164-15-974.

Papadopoulou E, Little HA, Hammar SA, Grumet R. 2005.Effect of modified endogenous ethylene production on sex expression, bisexual flower development and fruit production inmelon (Cucumis melo L.).Sexual Plant Reproduction. 18:131-142. https://doi:10.1007/s00497-005-0006-0.

Renner SS. 2014. The relative and absolute frequencies of angiosperm sexual systems: dioecy, monoecy, gynodioecy, and an updated online database. American journal of botany.101:1588-1596. https://doi:10.3732/ajb.1400196. 
516 Shimakawa A, Shiraya T, Ishizuka Y, Wada KC, Mitsui T, Takeno K. 2012. Salicylic acid is
517 involved in the regulation of starvation stress-induced flowering in Lemna paucicostata.

517

518

519

520

521

522

523

524

525

526

527

528

529

530

531

532

533

534

535

536

537

538

539

540

541

542

543

544

545

546

547

548

549

550

551

552

553

554

555 Journal of plant physiology. 169:987-991. https://doi:10.1016/j.jplph.2012.02.009.

Sun S, Verdu M. 2016. Evolutionary correlation of heterodichogamy and floral longevity. Evolutionary Ecology. 30 (6)1023-1030. https://doi:10.1007/s10682-016-9865-0.

Tal O. 2009. Acer pseudoplatanus (Sapindaceae): Helerodichogamy and thrips pollination. Plant systematics and evolution.278(3-4): 211-221. https://doi:10.1007/s00606-008-0141-9.

Tanurdzic M, Banks JA. 2004. Sex-determining mechanisms in land plants. The Plant Cell.16:61-71.

Teichert H, Dötterl S, Gottsberger G. 2011. Heterodichogamy and nitidulid beetle pollination in Anaxagorea prinoides, an early divergent Annonaceae. Plant Systematics and Evolution. 291: 25-33. https://doi:10.1007/s00606-010-0367-1.

Teo ZWN, Song S, Wang Y, Liu J, Yu H. 2014. New insights into the regulation of inflorescence architecture. Trends in plant science. 19, 158-165. https://doi:10.1016/j.tplants.2013.11.001.

Wada KC, Yamada M, Shiraya T, Takeno K. 2010. Salicylic acid and the flowering gene FLOWERING LOCUS T homolog are involved in poor-nutrition stress-induced flowering of Pharbitis nil. Journal of Plant Physiology. 167(6): 447-452. https://doi:10.1016/j.jplph.2009.10.006.

Wang N. 2019. Expression Analysis of the Gene in Tobacco BR Signaling Pathway and Flowerrelated in Flower Bud Differentiation in Response to Low Temperature in Seedling Stage. Southwest University.

Wang Q, Zeng G. 1997.Inducing Singal of Sex Differentiation in Higher Plant. Plant physiology communication. 33(2):147- 151. https://doi:10.13592/j.cnki.ppj.1997.02.027.

Wang Q, Zeng G. 1996. Progress of Study on Sexual Differentiation of Monoecious Plants in vitro. Plant physiology communication. 32(5):385-389. https://doi:10.13592/j.cnki.ppj.1996.05.025.

Wang X. 2001. Study on the dichogamy of Juglans mandshurica maxim. Bulletin of botanical research. 21(3):388-391.

Wang X, Zhang P, Du Q, He H, Zhao L, Ren Y, Endress PK. 2012.Heterodichogamy in Kingdonia (Circaeasteraceae, Ranunculales). Annals of botany. 109:1125-1132. https://doi:10.1093/aob/mcs041.

Weiler EW, Jourdan PS, Conrad W. 1981.Levels of indole-3-aceticacid in intact and decapitated coleoptiles as determined by a specic and highly sensitive solid-phase enzyme immunoassay. Planta, 153(6) : 561- 571.

Wu T, Qin Z, Zhou X, Feng Z, Du Y. 2010.Transcriptom profile analysis of floral sex determination in cucumber. Journal of plant physiology. 167: 905-913. https://doi:10.1016/j.jplph.2010.02.004.

Xing H. 2007. The Basic Studies on Sexual Determlnation and Related Gene in Ramie (Boehmeria Nivea). Chinese Academy of Agricultural Sciences.

Peer) reviewing PDF | (2020:12:56792:7:0:NEW 13 Sep 2021) 
556

557

558

559

560

561

562

563

564

565

566

567

568

569

570

571

572

573

574

575

576

577

578

579

580

581

582

583

584

585

586

587

588

589

590

591

592

593

594

595
Xing L, Zhang D, Li Y, Shen Y, Zhao X, Ma J, An N, Han M. 2015. Transcription profles reveal sugar and hormone signaling pathways mediating flower induction in apple (Malus domestica Borkh.). Plant Cell Physiol. 56:2052-2068. https://doi.org/10.1093/pcp/pcv124.

Xing L, Zhang D, Song X, Weng K, Shen Y, Li Y, Zhao C, Ma J, An N, Han M. 2016. Genome-wide sequence variation identifcation and floral-associated trait comparisons based on there-sequencing of the 'Nagafu No. 2' and 'Qinguan' varieties of apple (Malus domestica Borkh.). Crop Sci Hortic. 7: 908. https://doi:10.3389/fpls.2016.00908.

Yamaguchi N, Winter CM, Wu M, Kanno Y, Yamaguchi Y, Seo M, Wagner D. 2014. Gibberellin acts positively then negatively to control on set of flower formation in Arabidopsis. Science. 344: 638-641.

Yu D, Li L, Wei H, Yu S. 2020.Identifcation and profling of microRNAs and diferentially expressed genes during anther development between a genetic male-sterile mutant and its wildtype cotton via high-throughput RNA sequencing. Molecular Genetics and Genomics. https://doi.org/10.1007/s00438-020-01656-y.

Zhang L, Guo C, Qin B, Lu X, Yang Y, Qi Y, Shen H. 2019b.Phonological Characteristics of Flowering and Pollen Viability of Juglans mandshurica. Journal of Northeast Forestry University. 47(5):4-8. https://doi:10.13759/j.cnki.dlxb.2019.05.002.

Zhang L, Qin B, Guo C, Lu X, Yang Y, Qi Y, Yu G, Zhu R. 2019a.Study on Changes of Material Metabolism during Flower Bud Formation of Juglans mandshurica. Journal of Shenyang Agricultural University. 50(3):280-288. https://doi:10.3969/j.issn.10001700.2019.03.004.

Zhong H, Qin Z. 2010. Effect of plant growth substances on sex differentiation of cucumber. China Vegetables. 1(6): 1-7.

Zhu Z, Jiang C, Shi Y, ChenW, Chen N, Zhao M, Wu W. 2015. Variations of Endogenous Hormones in Lateral Buds of Olive Trees(Olea europaea) during Floral Induction and Flower-Bud Differentiation. Scientia Silvae Sinicae. 51(11):32-39. https://doi:10.11707 / j.1001-7488.20151105. 


\section{Figures and captions}

597 Figure 1. Male and female flower buds of Juglans mandshurica

598 Figure 2. Length distribution of Unigene for transcriptome in Juglans mandshurica

599 Figure 3. EggNOG function classification of Unigene for transcriptome in Juglans mandshurica

600 Figure 4. GO annotation analysis of T1vsT2

601 Figure. 5. KEGG pathway enrichment of T1vsT2

602 Figure 6. GO annotation analysis of M1vsM2

603 Figure7. KEGG pathway enrichment of M1vsM2

604 Figure 8. Number of differentially expressed genes in male and female flower buds

605 Figure 9. The differential expressed transcription factors between the male and female flower buds

606 Figure 10. Validation of 12 randomly selected differentially expressed genes (DEGs) derived from RNA-

607 seq using Real-time quantitative RT-PCR

608

609 Figure (S1a). E-value distribution of BLAST hits for each unigene

610 Figure (S1b). Similarity distribution of the top BLAST hits for each unigene

611 Figure (S1c) Species distribution of the top BLAST hits

612 Figure S2. GO function annotion and classification of Unigene for transcriptome in Juglans mandshurica

613 Figure S3. KEGG classification of Unigene for transcriptome in Juglans mandshurica

614 


\section{Figure 1}

Figure 1. Male and female flower buds of Juglans mandshurica.

(A)
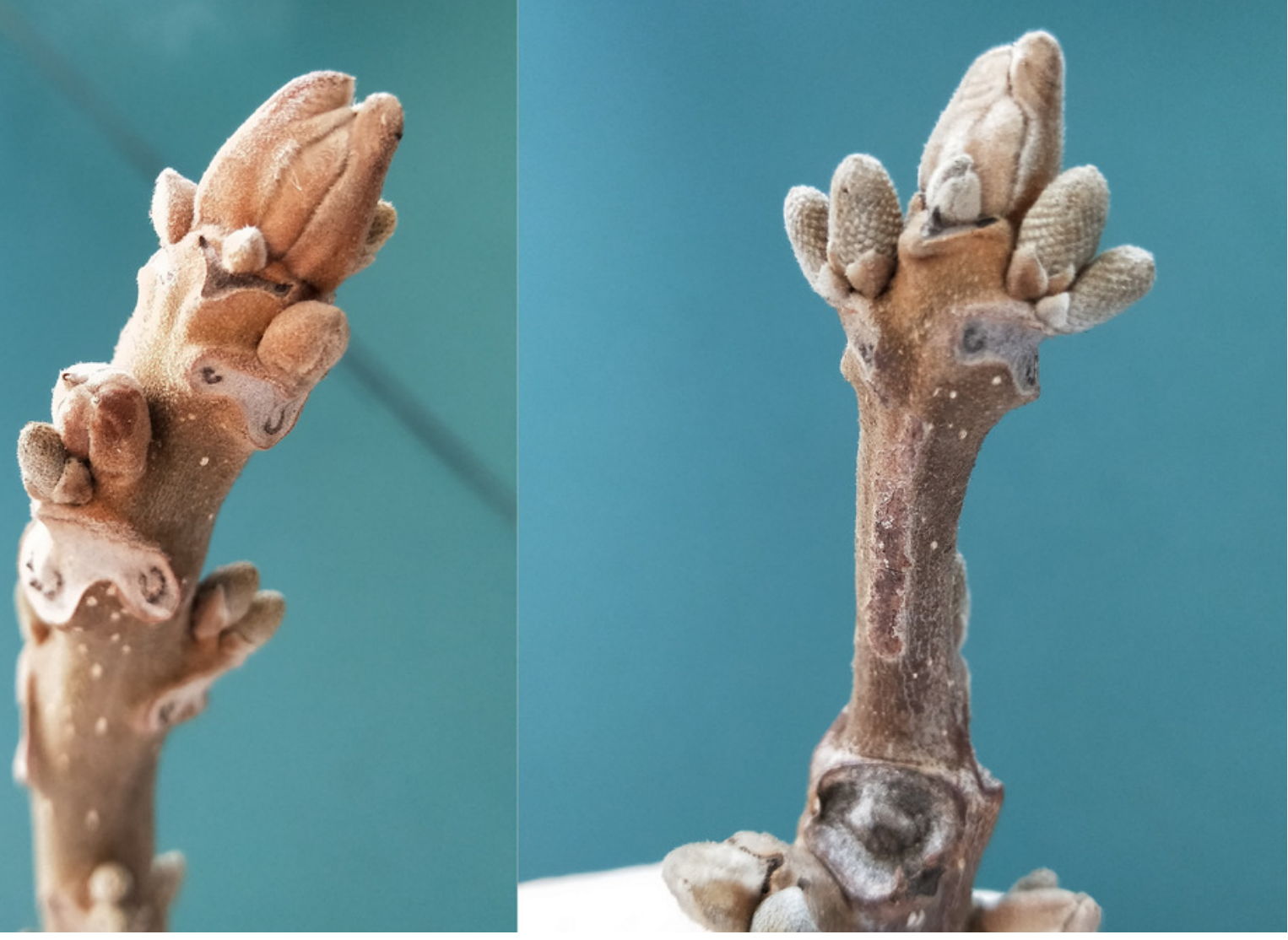
Figure 2

Length distribution of unigene for transcriptome in J. mandshurica.

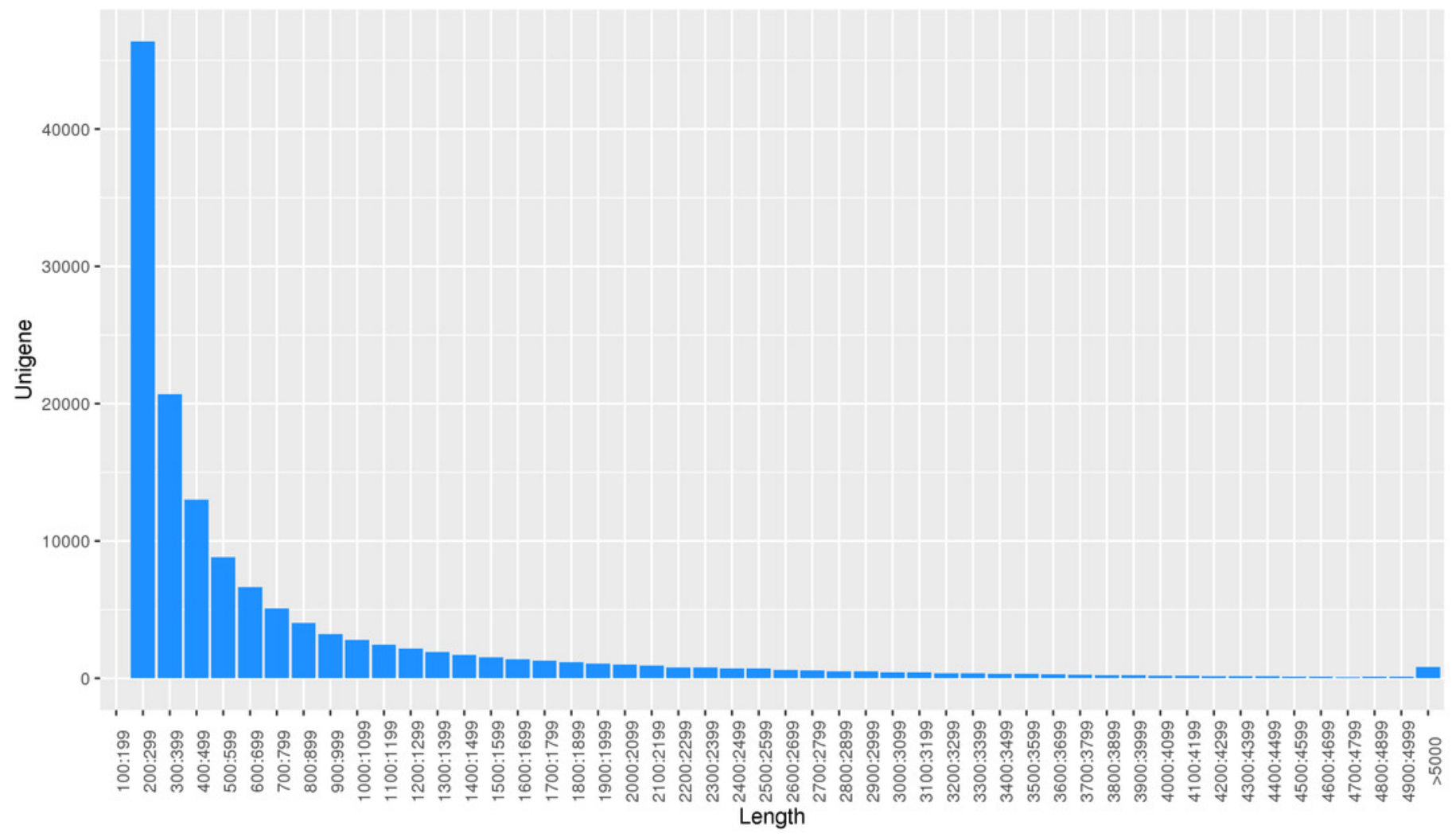


Figure 3

EggNOG function classification of unigene for transcriptome in J. mandshurica.

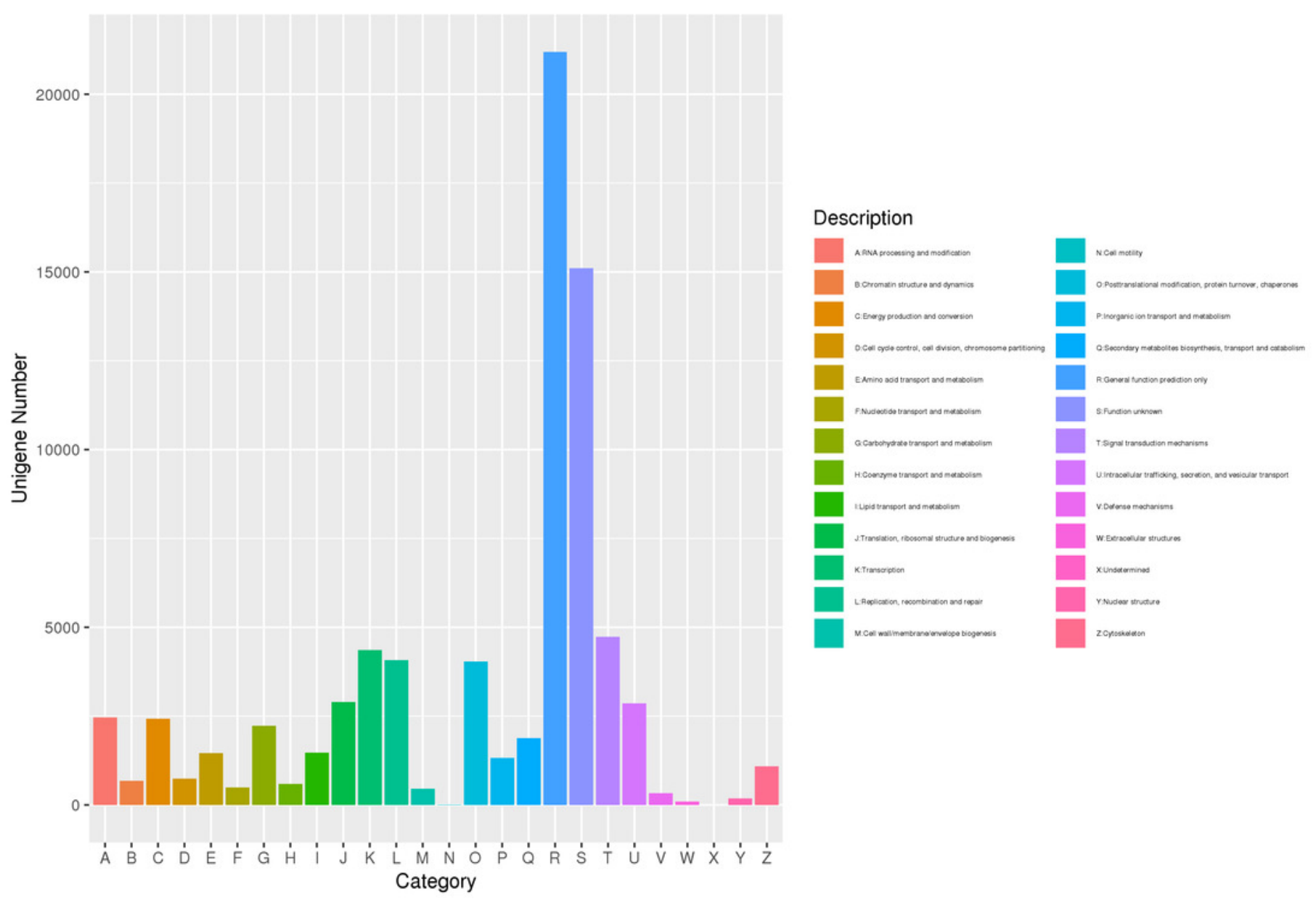


Figure 4

Figure 4.GO annotation analysis between male and female flower buds of female precursor Juglans mandshurica (T1vsT2)

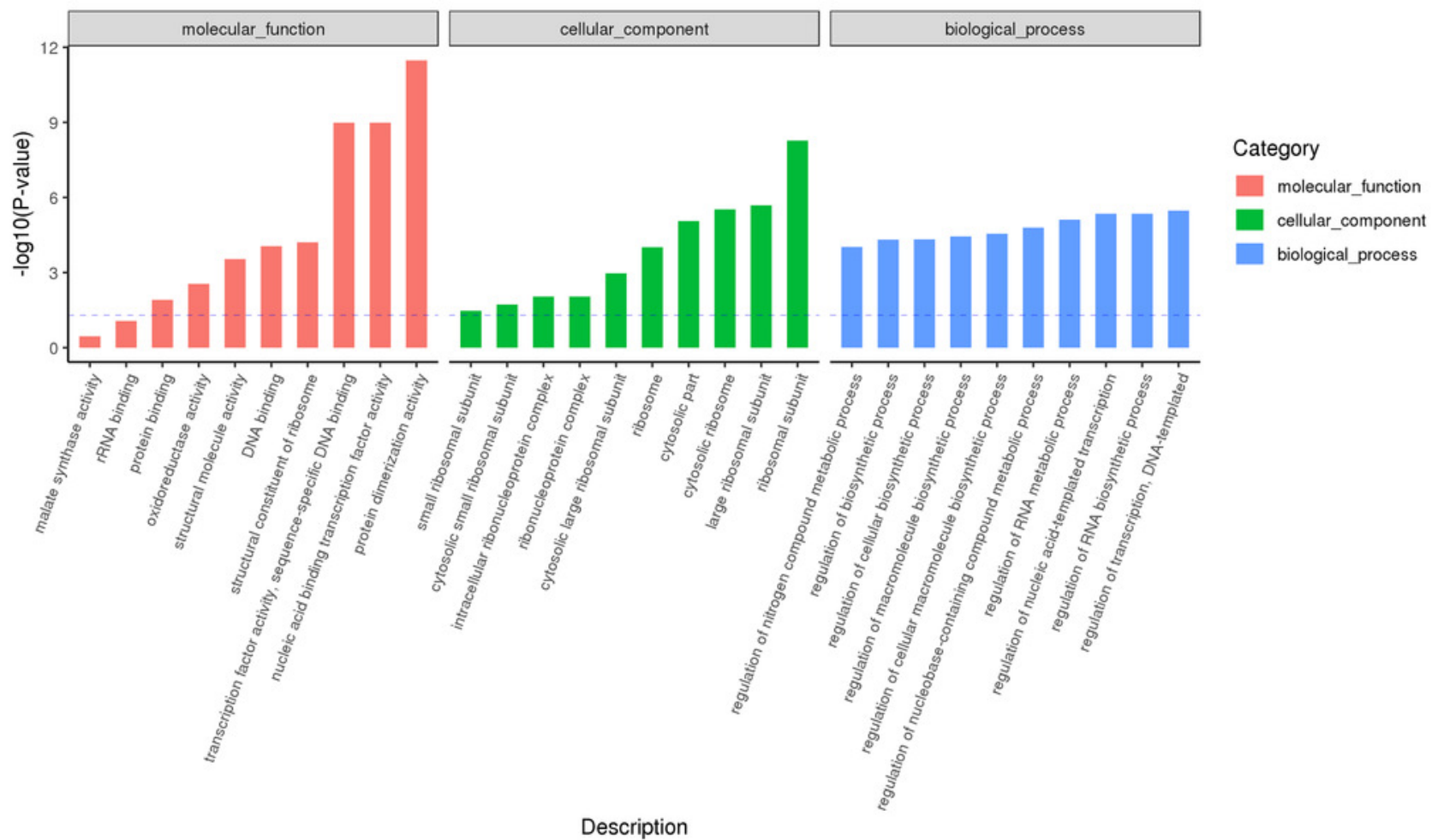


Figure 5

Figure 5.KEGG pathway enrichment of female and male flower buds of female precursor J. mandshurica (T1vsT2).

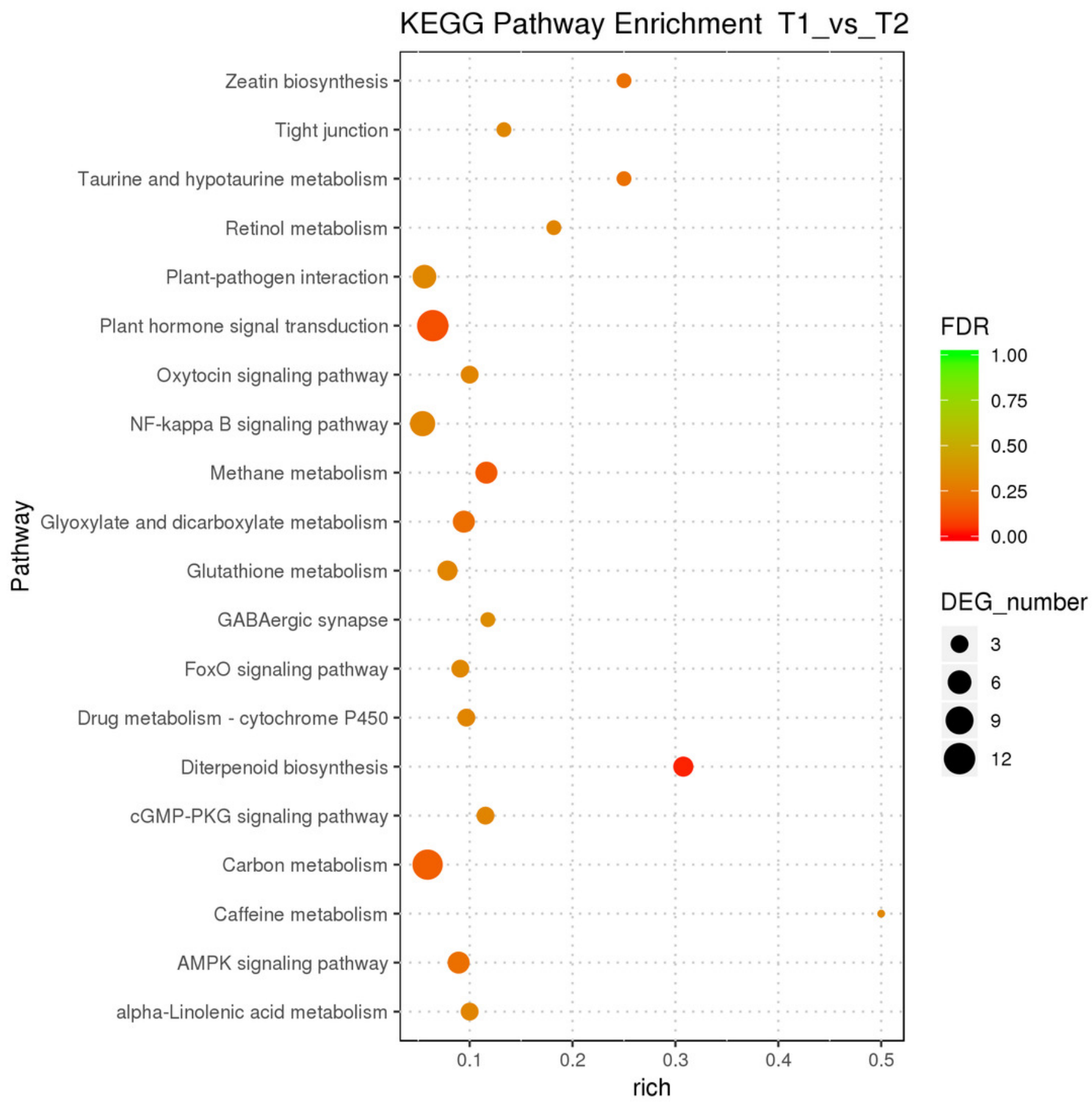


Figure 6

Figure 6. $\mathrm{GO}$ annotation analysis between male and female flower buds of male precursor Juglans mandshurica (M1vsM2).

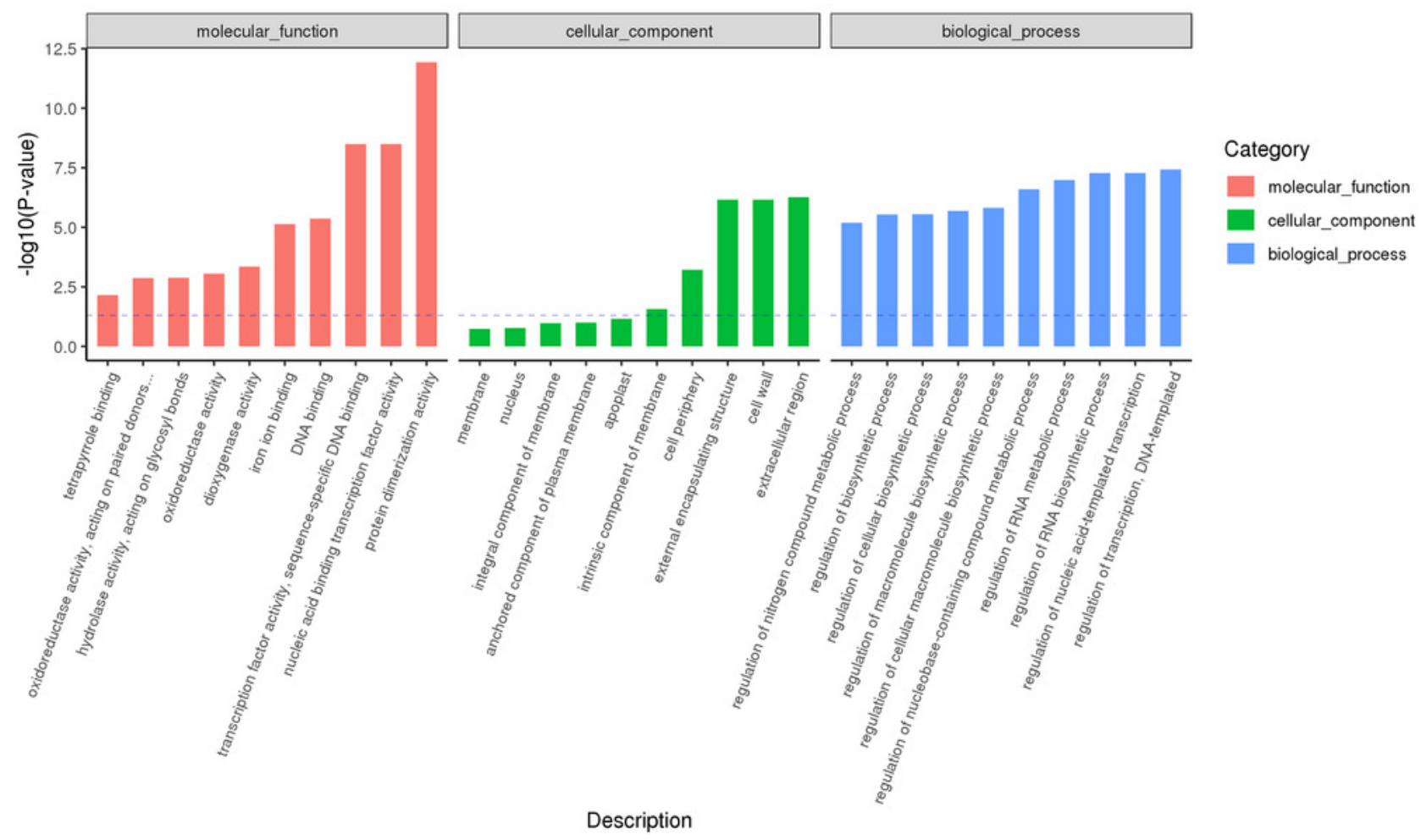




\section{Figure 7}

\section{Figure 7.KEGG pathway enrichment of female and male flower buds of male precursor J. mandshurica (M1vsM2).}

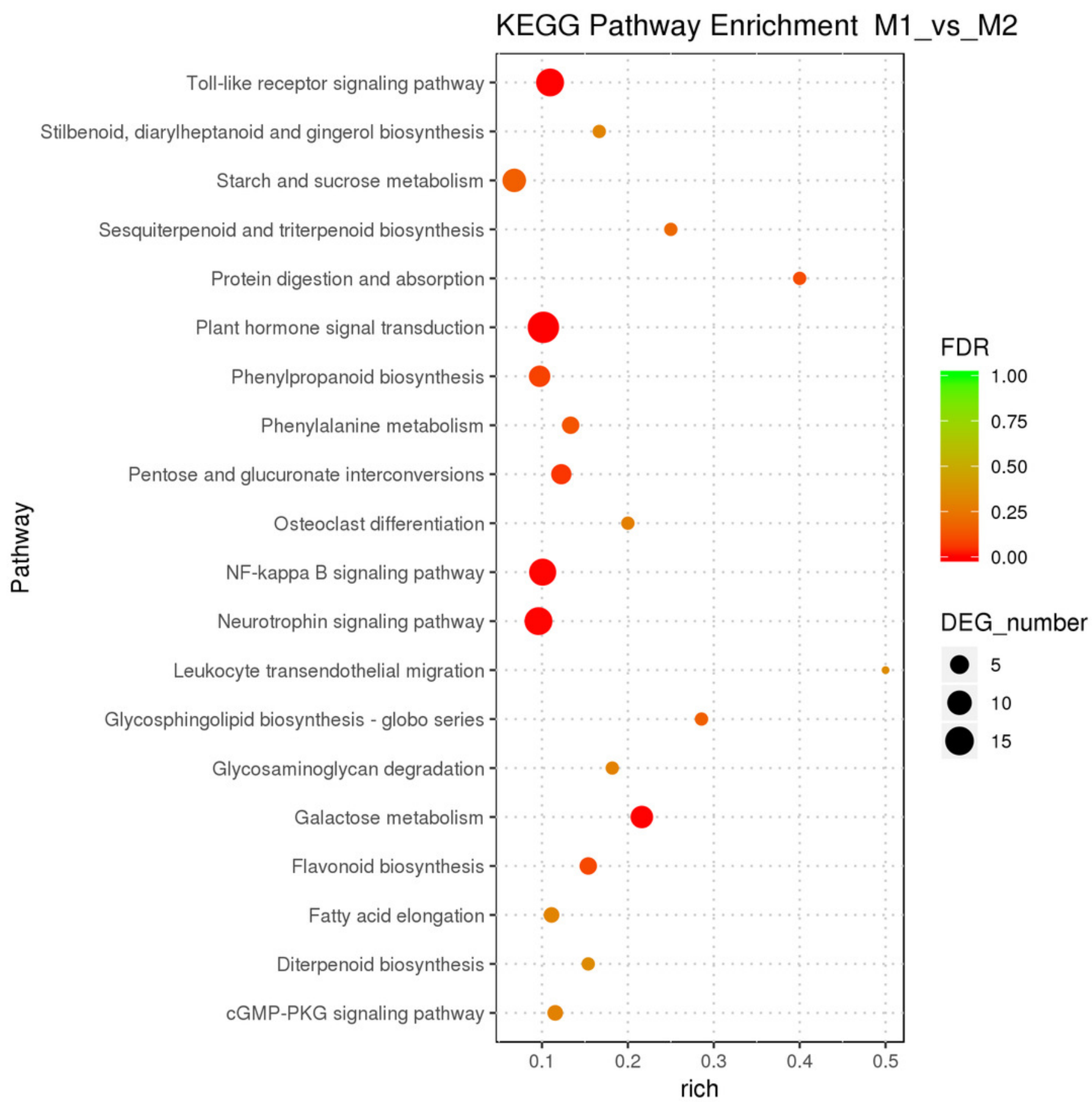


Figure 8

Figure 8. Number of differentially expressed genes in male and female flower buds(A stands for IAA related differentially expressed transcription factors, B stands for ETH related differentially expressed transcription factors)
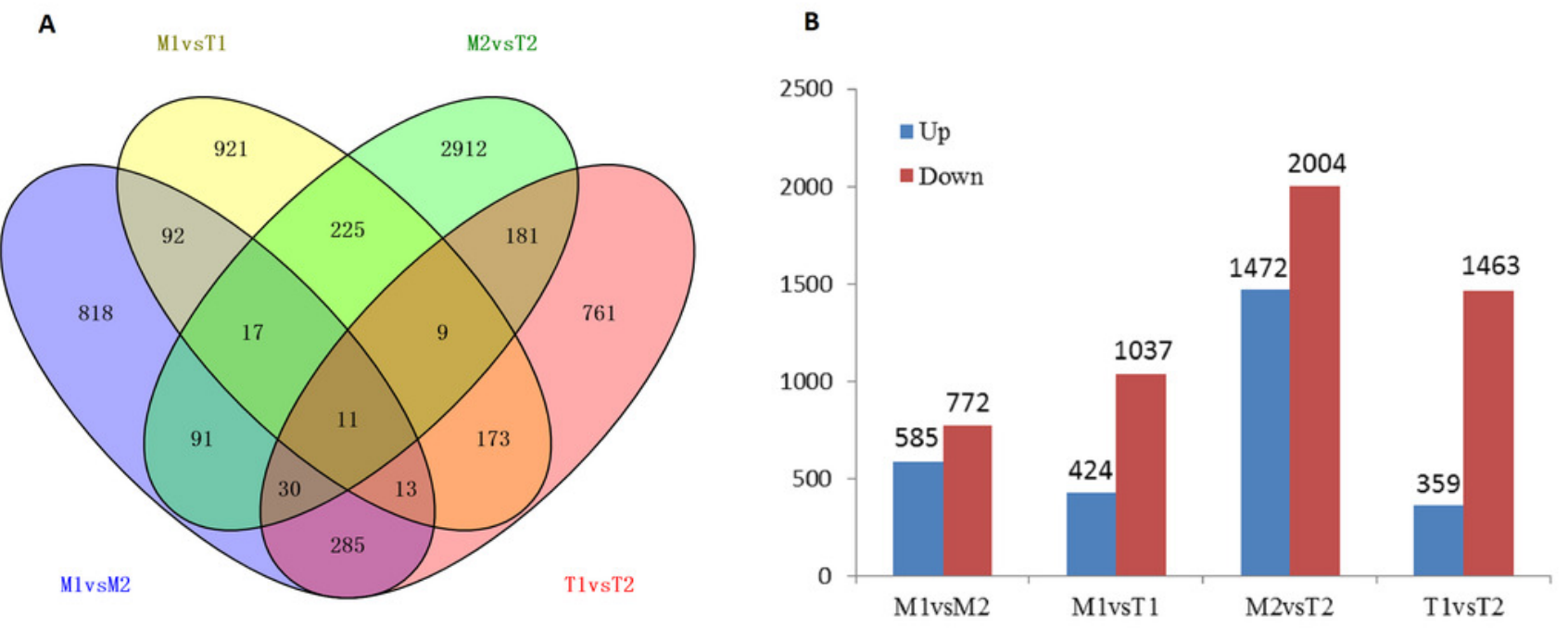


\section{Figure 9}

Figure 9.The differential expressed transcription factors between the male and female flower buds

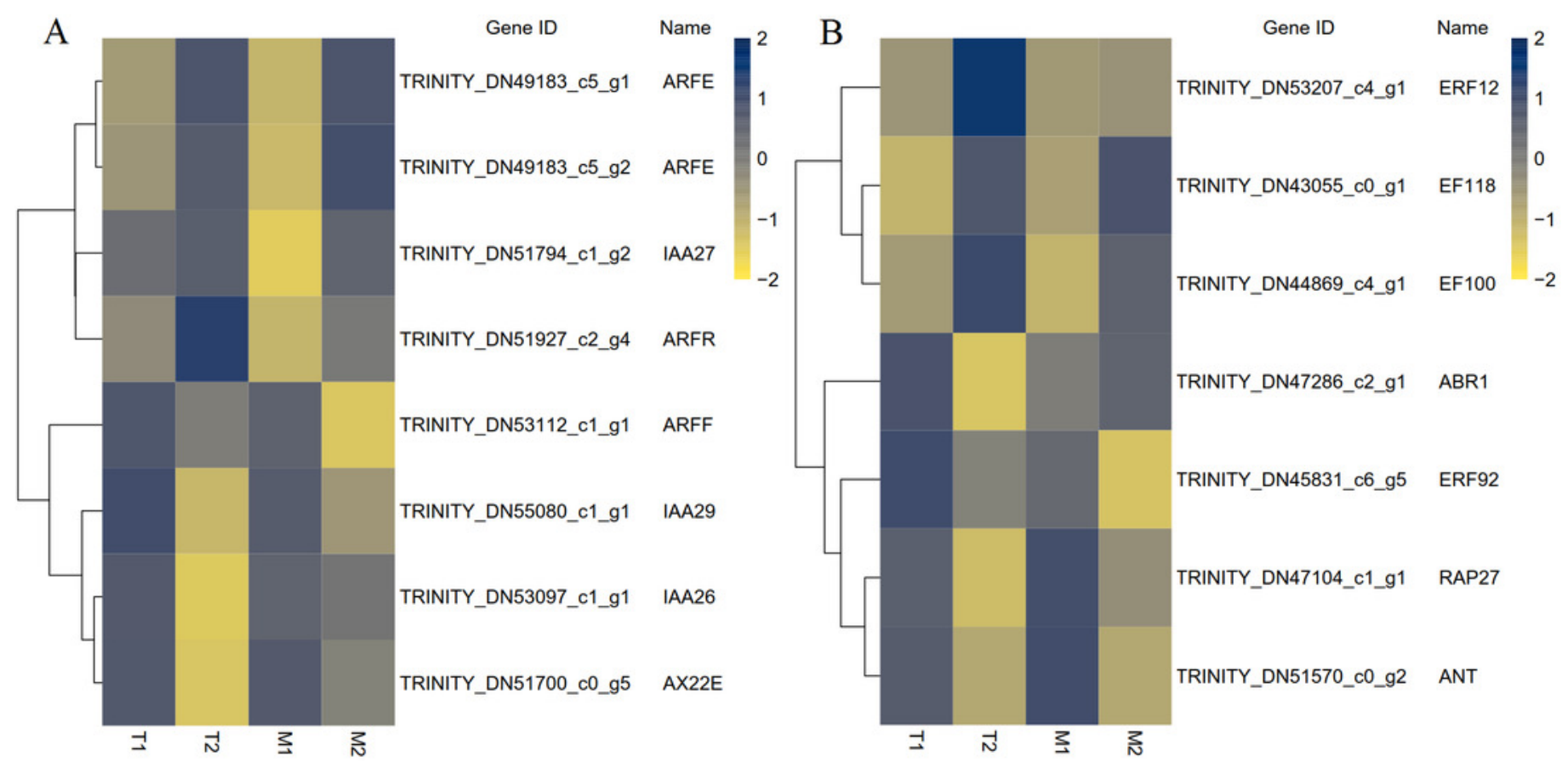


Figure 10

Validation of 12 randomly selected differentially expressed genes (DEGs) derived from RNA-seq using qRT-PCR. 
A

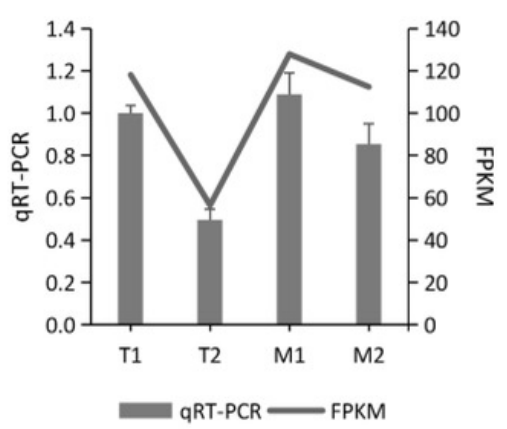

D
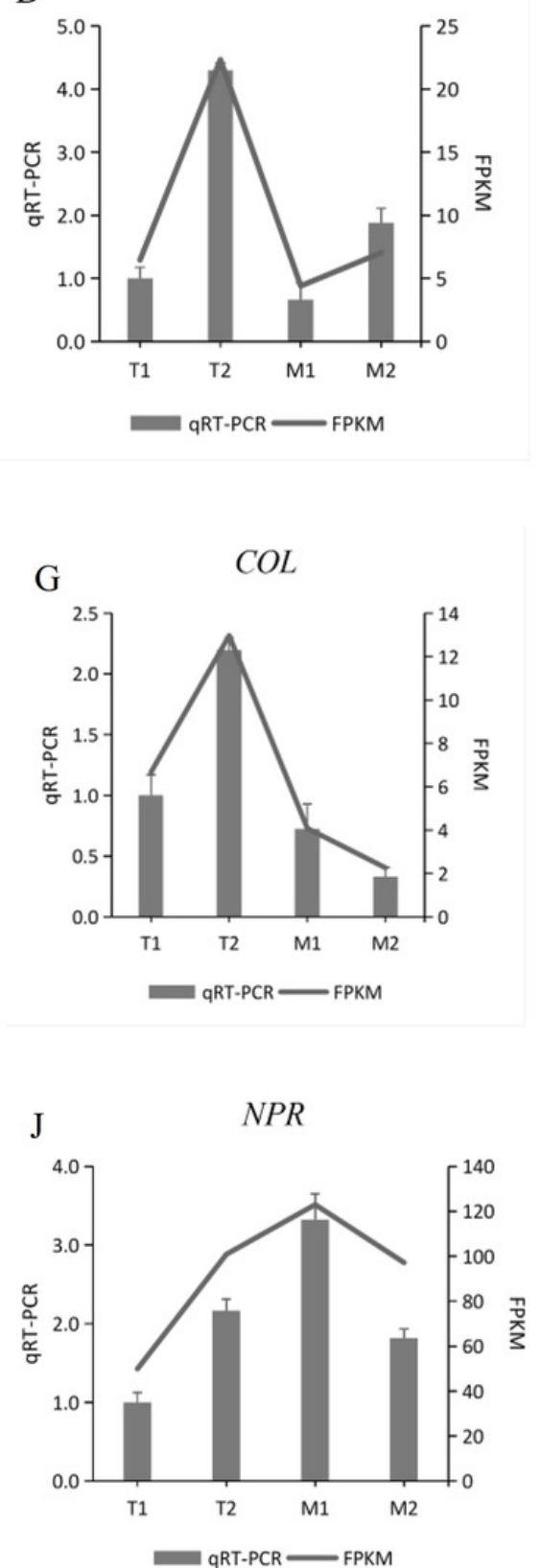

B

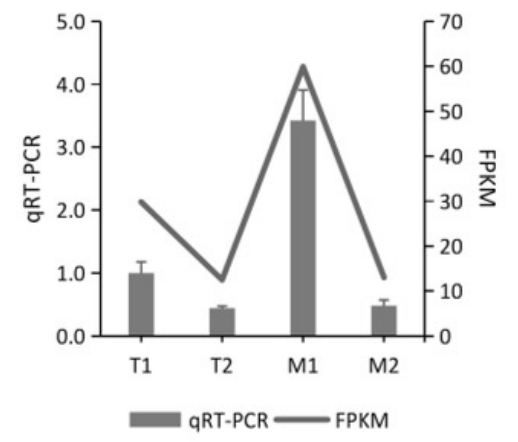

E

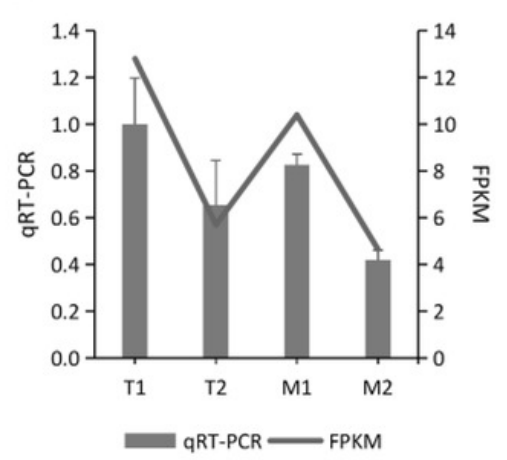

$\mathrm{H}$

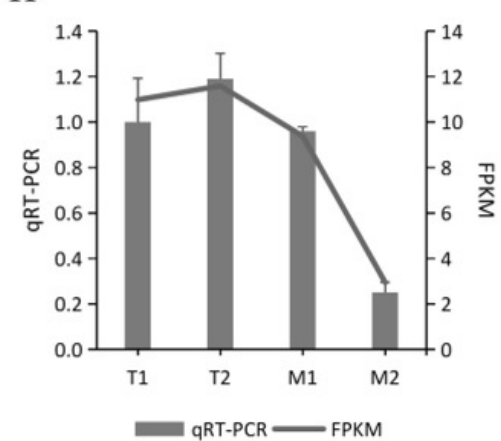

K

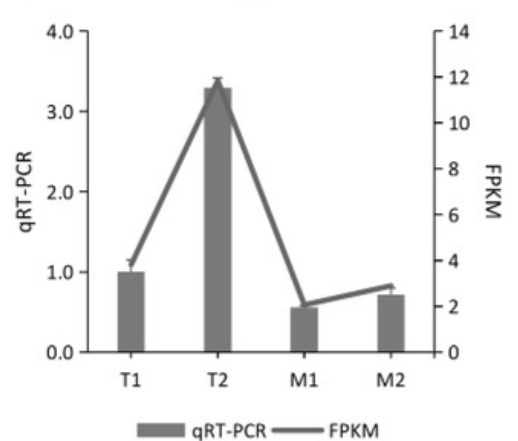

C $\quad A R F$

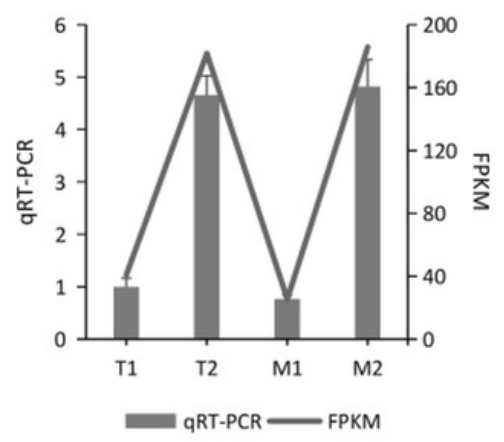

F

$E R F$

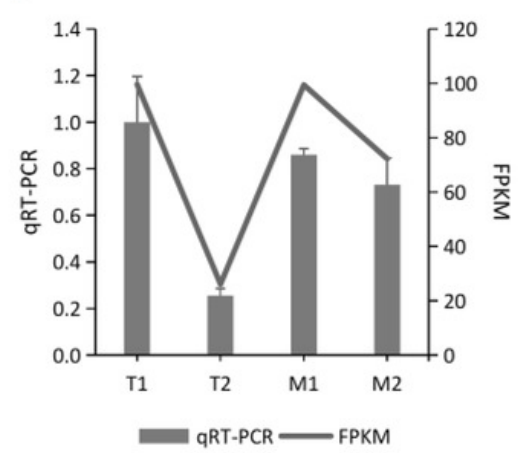

I

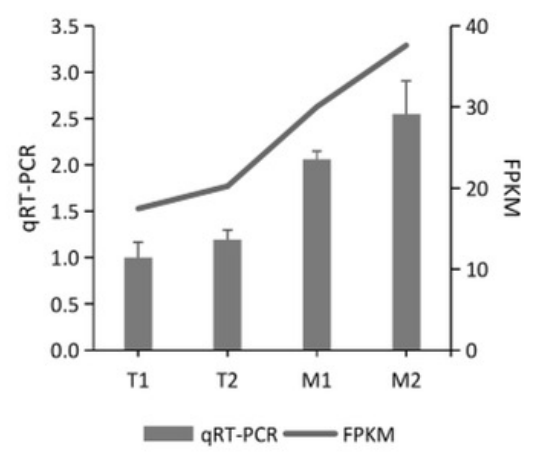

$\mathrm{L}$

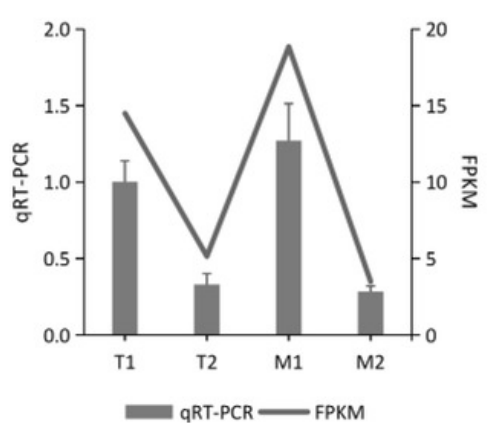




\section{Table 1 (on next page)}

Notes on unigene function of Juglans mandshurica transcriptome 
1

Table 1. Notes on unigene function of Juglans mandshurica transcriptome.

\begin{tabular}{ccccccc}
\hline Database & NR & GO & KEGG & EggNOG & Swissprot & In all database \\
\hline Number & 60415 & 29402 & 6845 & 57250 & 45347 & 5312 \\
Percentage(\%) & 43.74 & 21.28 & 4.96 & 41.44 & 32.83 & 3.85 \\
\hline
\end{tabular}

2 
Table 2 (on next page)

Plant hormone content of J. mandshurica flower buds at different stages 
Table 2. Plant hormone content of $J$. mandshurica flower buds at different stages

\begin{tabular}{|c|c|c|c|c|c|c|}
\hline \multirow{2}{*}{ Hormone } & \multirow{2}{*}{ Sample } & \multicolumn{5}{|c|}{ Date } \\
\hline & & 3.17 & 3.25 & 4.02 & 4.10 & 4.15 \\
\hline \multirow{4}{*}{$\begin{array}{c}\text { IAA } \\
(\mu \mathrm{g} / \mathrm{g})\end{array}$} & $\mathrm{T} 1$ & $18.17 \pm 1.16 \mathrm{ABb}$ & $20.79 \pm 0.28 \mathrm{Aa}$ & $25.05 \pm 1.21 \mathrm{Ab}$ & $32.11 \pm 2.39 \mathrm{Aa}$ & $28.78 \pm 1.25 \mathrm{Bb}$ \\
\hline & $\mathrm{T} 2$ & $17.14 \pm 1.43 \mathrm{Bb}$ & $16.62 \pm 0.76 \mathrm{Bb}$ & $27.59 \pm 0.44 \mathrm{Aa}$ & $26.32 \pm 1.19 \mathrm{Bb}$ & $39.03 \pm 1.31 \mathrm{Aa}$ \\
\hline & M1 & $21.06 \pm 0.88 \mathrm{Aa}$ & $20.14 \pm 0.16 \mathrm{Aa}$ & $25.23 \pm 1.47 \mathrm{Ab}$ & $31.41 \pm 0.85 \mathrm{Aa}$ & $30.50 \pm 1.64 \mathrm{Bb}$ \\
\hline & M2 & $20.59 \pm 0.87 \mathrm{Aa}$ & $16.55 \pm 0.38 \mathrm{Bb}$ & $24.38 \pm 1.03 \mathrm{Ab}$ & $27.78 \pm 1.63 \mathrm{ABb}$ & $31.43 \pm 1.49 \mathrm{Bb}$ \\
\hline \multirow{4}{*}{$\begin{array}{c}\text { CTK } \\
(\mathrm{ng} / \mathrm{g})\end{array}$} & $\mathrm{T} 1$ & $151.42 \pm 5.40 \mathrm{Bb}$ & $246.86 \pm 14.33 \mathrm{Ab}$ & $309.37 \pm 13.49 \mathrm{Aab}$ & $260.66 \pm 12.97 \mathrm{Aab}$ & $355.08 \pm 11.45 \mathrm{Aa}$ \\
\hline & $\mathrm{T} 2$ & $180.37 \pm 4.13 \mathrm{Aa}$ & $275.90 \pm 10.53 \mathrm{Aa}$ & $320.71 \pm 5.52 \mathrm{Aa}$ & $245.70 \pm 12.32 \mathrm{Ab}$ & $284.82 \pm 11.36 \mathrm{Bc}$ \\
\hline & M1 & $114.23 \pm 9.79 \mathrm{Cc}$ & $163.65 \pm 7.30 \mathrm{Bc}$ & $296.69 \pm 10.18 \mathrm{Ab}$ & $278.77 \pm 11.92 \mathrm{Aa}$ & $347.64 \pm 18.50 \mathrm{Aa}$ \\
\hline & M2 & $126.46 \pm 9.83 \mathrm{Cc}$ & $253.05 \pm 11.43 \mathrm{Ab}$ & $254.93 \pm 12.34 \mathrm{Bc}$ & $266.40 \pm 8.15 \mathrm{Aab}$ & $319.14 \pm 14.88 \mathrm{ABb}$ \\
\hline \multirow{4}{*}{$\begin{array}{c}\text { GA } \\
(\mu \mathrm{g} / \mathrm{g})\end{array}$} & $\mathrm{T} 1$ & $0.29 \pm 0.005 \mathrm{Aa}$ & $0.26 \pm 0.003 \mathrm{Bc}$ & $0.31 \pm 0.03 \mathrm{Aa}$ & $0.36 \pm 0.03 \mathrm{Bc}$ & $0.37 \pm 0.02 \mathrm{Aa}$ \\
\hline & $\mathrm{T} 2$ & $0.25 \pm 0.01 \mathrm{Bb}$ & $0.32 \pm 0.01 \mathrm{Aab}$ & $0.31 \pm 0.02 \mathrm{Aa}$ & $0.41 \pm 0.02 \mathrm{ABab}$ & $0.33 \pm 0.00 \mathrm{BCb}$ \\
\hline & M1 & $0.20 \pm 0.02 \mathrm{Cc}$ & $0.33 \pm 0.02 \mathrm{Aa}$ & $0.35 \pm 0.01 \mathrm{Aa}$ & $0.38 \pm 0.01 \mathrm{ABbc}$ & $0.31 \pm 0.01 \mathrm{Cb}$ \\
\hline & M2 & $0.22 \pm 0.01 \mathrm{Cc}$ & $0.30 \pm 0.02 \mathrm{Ab}$ & $0.34 \pm 0.01 \mathrm{Aa}$ & $0.44 \pm 0.01 \mathrm{Aa}$ & $0.36 \pm 0.01 \mathrm{ABa}$ \\
\hline \multirow{4}{*}{$\begin{array}{c}\text { ETH } \\
(\mu \mathrm{g} / \mathrm{g})\end{array}$} & $\mathrm{T} 1$ & $151.81 \pm 10.24 \mathrm{Aa}$ & $105.50 \pm 10.46 \mathrm{Bc}$ & $104.90 \pm 7.35 \mathrm{Aa}$ & $175.75 \pm 9.73 \mathrm{Cc}$ & $300.46 \pm 14.14 \mathrm{Aa}$ \\
\hline & $\mathrm{T} 2$ & $117.94 \pm 2.51 \mathrm{Bb}$ & $119.73 \pm 2.78 \mathrm{ABb}$ & $115.94 \pm 8.89 \mathrm{Aa}$ & $246.95 \pm 13.55 \mathrm{Aa}$ & $273.75 \pm 8.71 \mathrm{Bb}$ \\
\hline & M1 & $139.24 \pm 3.33 \mathrm{Aa}$ & $132.30 \pm 5.82 \mathrm{Aa}$ & $115.89 \pm 10.15 \mathrm{Aa}$ & $193.18 \pm 5.82 \mathrm{BCbc}$ & $232.55 \pm 6.02 \mathrm{Cc}$ \\
\hline & M2 & $147.04 \pm 9.30 \mathrm{Aa}$ & $75.93 \pm 2.24 \mathrm{Cd}$ & $115.00 \pm 7.07 \mathrm{Aa}$ & $211.25 \pm 7.83 \mathrm{Bb}$ & $210.22 \pm 6.46 \mathrm{Cd}$ \\
\hline \multirow{4}{*}{$\begin{array}{c}\text { BR } \\
(\mu \mathrm{g} / \mathrm{g})\end{array}$} & $\mathrm{T} 1$ & $0.39 \pm 0.02 \mathrm{Aa}$ & $0.40 \pm 0.02 \mathrm{BCb}$ & $0.49 \pm 0.01 \mathrm{Bb}$ & $0.54 \pm 0.04 \mathrm{Aa}$ & $0.62 \pm 0.03 \mathrm{Aa}$ \\
\hline & $\mathrm{T} 2$ & $0.34 \pm 0.02 \mathrm{Aa}$ & $0.35 \pm 0.03 \mathrm{Cc}$ & $0.49 \pm 0.03 \mathrm{Bb}$ & $0.56 \pm 0.04 \mathrm{Aa}$ & $0.60 \pm 0.05 \mathrm{Aab}$ \\
\hline & M1 & $0.39 \pm 0.02 \mathrm{Aa}$ & $0.49 \pm 0.02 \mathrm{Aa}$ & $0.50 \pm 0.01 \mathrm{Bb}$ & $0.55 \pm 0.05 \mathrm{Aa}$ & $0.54 \pm 0.04 \mathrm{Ab}$ \\
\hline & M2 & $0.37 \pm 0.04 \mathrm{Aa}$ & $0.43 \pm 0.02 \mathrm{Bb}$ & $0.56 \pm 0.03 \mathrm{Aa}$ & $0.55 \pm 0.04 \mathrm{Aa}$ & $0.56 \pm 0.01 \mathrm{Aab}$ \\
\hline \multirow{4}{*}{$\begin{array}{l}\text { ABA } \\
(n g / g)\end{array}$} & $\mathrm{T} 1$ & $203.23 \pm 17.11 \mathrm{ABa}$ & $129.33 \pm 14.13 \mathrm{Aa}$ & $158.68 \pm 7.79 \mathrm{Aa}$ & $292.41 \pm 22.64 \mathrm{Bb}$ & $377.00 \pm 21.01 \mathrm{ABa}$ \\
\hline & $\mathrm{T} 2$ & $101.49 \pm 27.99 \mathrm{Cc}$ & $150.48 \pm 20.88 \mathrm{Aa}$ & $147.02 \pm 18.75 \mathrm{Aab}$ & $217.38 \pm 27.47 \mathrm{Cc}$ & $424.86 \pm 34.76 \mathrm{Aa}$ \\
\hline & M1 & $243.95 \pm 16.86 \mathrm{Aa}$ & $78.98 \pm 21.38 \mathrm{Bb}$ & $154.47 \pm 11.68 \mathrm{Aa}$ & $355.25 \pm 7.08 \mathrm{Aa}$ & $323.42 \pm 16.91 \mathrm{Bb}$ \\
\hline & M2 & $158.68 \pm 29.05 \mathrm{BCb}$ & $70.25 \pm 1.60 \mathrm{Bb}$ & $128.13 \pm 13.05 \mathrm{Ab}$ & $282.78 \pm 23.80 \mathrm{Bb}$ & $399.80 \pm 32.65 \mathrm{ABa}$ \\
\hline \multirow{4}{*}{$\begin{array}{c}\text { SA } \\
(\mu \mathrm{g} / \mathrm{g})\end{array}$} & $\mathrm{T} 1$ & $33.18 \pm 3.42 \mathrm{Bc}$ & $46.79 \pm 5.34 \mathrm{Aa}$ & $49.27 \pm 2.31 \mathrm{Aa}$ & $61.62 \pm 1.24 \mathrm{Aa}$ & $65.61 \pm 2.10 \mathrm{Bb}$ \\
\hline & $\mathrm{T} 2$ & $48.85 \pm 1.91 \mathrm{Aa}$ & $47.82 \pm 0.57 \mathrm{Aa}$ & $50.62 \pm 1.71 \mathrm{Aa}$ & $55.42 \pm 1.99 \mathrm{Bb}$ & $76.19 \pm 2.80 \mathrm{Aa}$ \\
\hline & M1 & $38.17 \pm 1.43 \mathrm{Bb}$ & $45.12 \pm 2.04 \mathrm{Aab}$ & $47.58 \pm 3.17 \mathrm{Aa}$ & $58.56 \pm 1.91 \mathrm{ABab}$ & $60.57 \pm 3.27 \mathrm{Bb}$ \\
\hline & M2 & $35.32 \pm 2.37 \mathrm{Bbc}$ & $37.55 \pm 1.92 \mathrm{Ab}$ & $46.06 \pm 3.65 \mathrm{Aa}$ & $61.24 \pm 2.38 \mathrm{Aa}$ & $78.22 \pm 5.07 \mathrm{Aa}$ \\
\hline \multirow{4}{*}{$\begin{array}{c}\mathrm{JA} \\
(\mathrm{ng} / \mathrm{g})\end{array}$} & $\mathrm{T} 1$ & $2.45 \pm 0.27 \mathrm{ABa}$ & $2.40 \pm 0.09 \mathrm{Ab}$ & $2.40 \pm 0.21 \mathrm{Bb}$ & $2.79 \pm 0.25 \mathrm{Bb}$ & $3.83 \pm 0.23 \mathrm{Ab}$ \\
\hline & $\mathrm{T} 2$ & $2.54 \pm 0.14 \mathrm{Aa}$ & $2.45 \pm 0.08 \mathrm{Ab}$ & $2.56 \pm 0.07 \mathrm{Bb}$ & $2.68 \pm 0.15 \mathrm{Bb}$ & $4.23 \pm 0.21 \mathrm{Aa}$ \\
\hline & M1 & $2.05 \pm 0.07 \mathrm{BCb}$ & $2.68 \pm 0.14 \mathrm{Aa}$ & $2.95 \pm 0.11 \mathrm{Aa}$ & $3.01 \pm 0.02 \mathrm{Bb}$ & $4.22 \pm 0.12 \mathrm{Aa}$ \\
\hline & M2 & $1.85 \pm 0.12 \mathrm{Cb}$ & $2.70 \pm 0.15 \mathrm{Aa}$ & $3.18 \pm 0.06 \mathrm{Aa}$ & $3.83 \pm 0.18 \mathrm{Aa}$ & $3.35 \pm 0.05 \mathrm{Bc}$ \\
\hline
\end{tabular}

